\title{
Solutions de macromolécules rigides : effets de paroi, de confinement et d'orientation par un écoulement
}

\author{
L. Auvray \\ Laboratoire de Physique de la Matière Condensée, Collège de France, 75231 Paris Cedex 05, France
}

(Reçu le 7 juillet 1980, accepté le 23 septembre 1980)

\begin{abstract}
Résumé. - Nous examinons le comportement de solutions diluées de «bâtons durs ", en présence de diverses perturbations extérieures :

a) Près d'une paroi plane agissant de façon purement stérique (pas d'adsorption), la concentration en monomères est réduite sur une distance $L$ ( $L$, longueur des bâtons). Nous évaluons le profil de concentration et l'énergie inter-
\end{abstract} faciale résultante.

b) Dans une solution ternaire, de bâtons de sphères et de solvant, avec, là aussi, des interactions purement géométriques, nous montrons que deux sphères s'attirent dès que leur distance est inférieure à la longueur $L$ d'un bâton. Cet effet peut déstabiliser une solution colloïdale de sphères.

c) Dans un pore tubulaire, la viscosité apparente d'une solution diluée de bâtons doit être réduite. $\left(\delta \eta \sim \delta \eta_{\text {bulk }}(D / L)^{2}, D\right.$ est le diamètre du pore. $D<L$.)

d) A l'entrée d'un pore, les bâtons peuvent être fortement alignés par l'écoulement convergent. Nous étudions qualitativement cet effet pour des solutions diluées et aussi semi-diluées. Pour ce dernier cas, où les molécules sont enchevêtrées, nous utilisons l'analyse de Doi et Edwards, qui mène à des effets orientationnels très augmentés.

\begin{abstract}
We examine the behaviour of dilute solutions of long hard rods in the presence of various external perturbations :

a) Near a purely repulsive (non absorbing) wall, the monomer concentration is reduced over a distance $L$ (where $L$ is the rod length). We evaluate the concentration profile and the resulting interfacial energy.

b) In a ternary solution composed of hard rods, hard spheres and solvent, we show that two spheres attract each other if their distance is smaller than the rod length. This effect can destabilize a colloidal solution of spheres.

c) In a cylindrical pore the apparent viscosity for a dilute solution of rods is shown to be reduced. $\left(\delta \eta \sim \delta \eta_{\text {bulk }}(D / L)^{2}\right.$, where $D$ is the pore diameter. $D<L$.)

d) Near the pore orifice, for rapid solvent flow, the rods become strongly aligned in the convergent flow. We study this effect qualitatively for the case of dilute and semi-dilute solutions. For the latter case, where the molecules are entangled, we utilize the analysis of Doi and Edwards, which leads to very enhanced orientational effects.
\end{abstract}

Introduction. - L'étude théorique des solutions de bâtons durs et rigides n'a pas toujours suscité autant d'attention que celle des polymères flexibles. Il y a plusieurs raisons pour cela :

i) La classe de matériaux utilisables est plus restreinte : certaines structures biologiques (virus de la mosaique du tabac); des polypeptides (le polybenzyl glutamate en phase hélice) de nombreux polyélectrolytes et certaines particules colloïdales d'oxydes métalliques $\left(\mathrm{V}_{2} \mathrm{O}_{5}\right)$..

ii) Les phénomènes attendus sont moins riches et les limitations pratiques sont nombreuses. Les interactions électrostatiques, la légère flexibilité des longues chaînes, la formation d'agrégats limitent les applications dans des conditions bien précises de $\mathrm{pH}$, de salinité, de température et de concentration.

iii) Les résultats théoriques sont probablement d'une moins grande universalité que ceux qui ont été obtenus pour les systèmes de chaînes flexibles neutres.

Certaines propriétés des solutions de bâtons durs sont cependant connues et interprétées depuis longtemps. La plus saillante est sans doute l'existence d'une transition nématique à concentration suffisamment élevée $\left(c_{\mathrm{n}} \sim\left(L^{2} d\right)^{-1} ; L\right.$ longueur, $d$ largeur 
du bâtonnet) induite par les effets de volume exclu. (L. Onsager [1], P. Flory [2].) Si les prédictions des références $[1,2]$ sont effectivement adéquates pour de longues molécules, dures et rigides [3], elles tombent en défaut pour des molécules plus courtes - les cristaux liquides « ordinaires » (P.A.A. par exemple) chez lesquelles d'autres interactions peuvent également jouer un rôle important [4].

Les molécules longues, minces et rigides ont aussi des propriétés dynamiques intéressantes $[5,6]$. Nous retiendrons notamment celles, prédites récemment par Doi et Edwards [7] liées aux enchevêtrements des molécules en solution semi-diluée. Ils montrent que le temps de diffusion rotationnelle d'un bâtonnet est beaucoup plus long qu'en solution diluée à cause d'un mécanisme analogue conceptuellement à la reptation des chaînes flexibles dans un fondu [8]. En solution diluée, le coefficient de diffusion rotationnel est :

$$
D_{\mathrm{ro}}=\tau_{\mathrm{or}}^{-1}=\frac{2}{3} \frac{T}{\eta_{\mathrm{s}} L^{3}} \ln \left(\frac{2 L}{d}\right) \quad[6],
$$

en solution semi-diluée $D_{\mathrm{r}}=D_{\mathrm{ro}}\left(c_{\mathrm{p}} L^{3}\right)^{-2}$. L'exposant de $c_{\mathrm{p}}:-2$ exprime que les collisions binaires entre bâtons sont prédominantes dans la diffusion; $\eta_{\mathrm{s}}$ est la viscosité du solvant, $c_{\mathrm{p}}$ la concentration en polymères, $T$ la température (en unité d'énergie). Ce temps a été mesuré [9] dans la relaxation de la biréfringence Kerr d'une solution de long virus macrophage (M-13) à concentration variable pour 2 longueurs différentes $(L=8000 \AA, L=15000 \AA)$. Il suit bien, en fonction de la concentration, une loi en $c_{\mathrm{p}}^{2}$, mais il n'a pas été possible d'étudier précisément la dépendance en longueur (à cause sans doute de la flexibilité des virus étudiés).

Dans le présent article, nous voulons arriver à une compréhension qualitative des propriétés des solutions de bâtonnets dans un milieu confiné (par exemple, un pore de dimension comparable à la longueur de la molécule). Nous supposons constamment que les contraintes sont uniquement géométriques : nous négligerons tout effet d'absorption, d'ancrage aux parois, de coincement (et de déformation) des molécules.

Un premier aspect concerne les propriétés interfaciales de la solution. Une paroi solide infiniment répulsive limite les orientations d'un bâtonnet situé dans son voisinage et repousse donc ceux-ci sur une distance de l'ordre de la longueur du bâton $(L)$. Quand les interactions entre bâtons sont négligeables (cas des solutions diluées), il se crée près de la paroi une zone de déplétion en monomères, $G$. Chauveteau [10] a récemment introduit cette idée, par analogie avec le cas des polymères flexibles $[8,11]$ pour expliquer la réduction de viscosité apparente d'une solution de xanthane (polysaccaride rigide) entraînée dans les nucléopores. Comme pour des polymères flexibles, cette zone de déplétion induit une interaction entre surfaces solides séparée par la solution [11]. Nous étudions cet effet pour des parois planes et sphériques (de rayon grand ou petit devant la longueur du bâton). Deux sphères s'attirent dès qu'elles se trouvent à une distance l'une de l'autre inférieure à la longueur du bâton, et cette attraction peut dans certains cas déstabiliser la suspension de sphères. Cela peut être un cas intéressant de flocculation (éventuellement anisotrope, si la solution est nématique) induite par un polymère $[11,12]$.

Le second aspect est dynamique et rhéologique. Nous évaluons la viscosité intrinsèque apparente de la solution diluée dans un tube de diamètre $D$ inférieur à $L$. Elle suit une loi d'échelle simple quand on néglige les interactions hydrodynamiques :

$$
\delta \eta_{\mathrm{conf}} \sim \delta \eta_{\mathrm{bulk}}\left(\frac{D}{L}\right)^{2}
$$

Nous étendons la discussion au cas des solutions diluées et nous discutons l'effet possible des enchevêtrements.

Le dernier aspect est plus pratique : nous examinons le comportement de la solution (diluée ou semi-diluée) dans un écoulement convergent vers l'entrée d'un pore cylindrique et les conditions de pénétration d'un bâton. En particulier, nous étudions l'orientation d'un bâtonnet dans un écoulement à gradient de vitesse longitudinal et nous étendons phénoménologiquement l'étude de Kramers [5] au cas des solutions semidiluées.

L'étude de telles situations a aussi un certain intérêt pratique :

a) En récupération assistée du pétrole [13], où l'on pousse le pétrole (dans un milieu poreux) par des solutions chargées en polyacrylamides ou polysaccharides. Si le polymère ne s'adsorbe pas sur les parois, la viscosité de l'eau devient comparable à celle des huiles lourdes et ceci permet à l'eau de déplacer le pétrole en évitant certaines instabilités hydrodynamiques [14] (pénétration en longs doigts du liquide le moins visqueux dans le liquide le plus visqueux sans entraîner celui-ci);

b) Comme méthode d'étude des filtres et des milieux poreux en général. Les géométries simples que nous décrivons ici ne se rencontrent que dans certaines situations spécialement préparées : par exemple, nucléopores percés dans une membrane de polycarbonate. Les milieux poreux naturels (grès, sable, craie), les verres poreux ont une structure plus complexe (distribution de taille de pore) qui peut aussi être sondée par les polymères;

c) Pour l'extrusion de fibres à haut module d'élasticité : polyamides aromatiques en solution dans l'acide sulfurique (Kevlar);

d) Dans l'étude de certaines solutions calloïdales.

Notre travail est surtout qualitatif. Beaucoup des effets sont numériquement complexes. Mais nous 
prévoyons certaines lois d'échelle (en fonction de la longueur des chaînes, ou de la concentration) qui pourraient être testées par des expériences systématiques.

Tout au long de l'article, $L$ désignera la longueur du bâton, $d$ sa largeur $(d \ll L), N$ l'indice de polymérisation de la molécule, $c$ la concentration (nb. de monomères par $\left.\mathrm{cm}^{3}\right)$ et $c_{\mathrm{p}}(=c / N)$ la concentration en polymère. Nous introduisons la concentration de recouvrement des bâtons $c_{\mathrm{p}}^{*}\left(c_{\mathrm{p}}^{*} \sim L^{-3}\right)$ qui sépare le régime dilué, où les interactions stériques entre macromolécules sont négligeables du régime semidilué $\left(c_{\mathrm{p}}^{*}<c<c_{\mathrm{pn}}\right), c_{\mathrm{pn}}$ est la concentration à la transition nématique $\left(c_{\mathrm{pn}} \sim\left(L^{2} d\right)^{-1}\right)$.

Nous nous intéresserons plus souvent à $c_{\mathrm{p}}<c_{\mathrm{p}}^{*}$ (sauf dans la section 3).

1. Effets interfaciaux dans une solution de bâtons durs. - Quand la solution est diluée et les bâtons minces $(d \ll L)$, les propriétés d'équilibre de la solution près d'une surface infiniment répulsive donnée ne dépendent que de la longueur du bâton et peuvent être déduites très simplement à partir d'arguments géométriques.

1.1 ZONE DE DÉPLÉTION EN MONOMÈRE PRÈS D'UNE SURFACE RÉPULSIVE. - Nous distinguerons deux cas limites selon que la surface s'étend sur des distances plus grandes ou plus petites que la taille de la molécule. Dans le premier cas (paroi plane), la longueur caractéristique de la zone de déplétion en monomère est $L$,

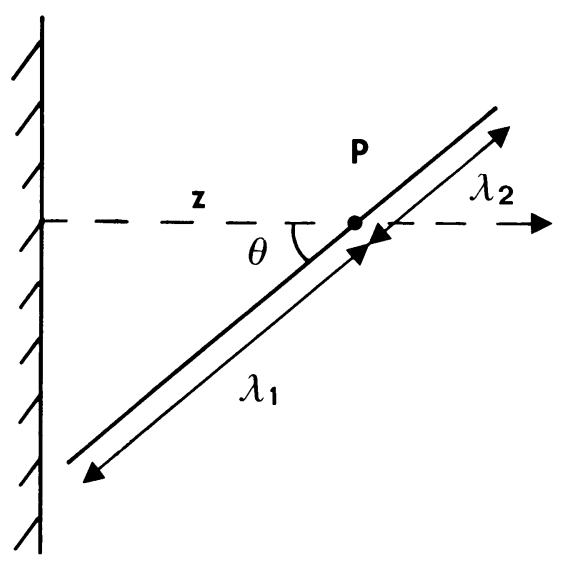

Fig. 1. - Configuration d'un bâtonnet près d'une paroi. Un monomère est fixé en $\mathrm{P}$, à une distance $z$ de la paroi et à des distances $\lambda_{1}$ et $\lambda_{2}$ des extrémités du bâton. Les probabilités relatives à de telles configurations sont :

$$
\begin{array}{ll}
\text { si } z>\lambda_{1}>\lambda_{2} & \mathfrak{T}_{\lambda}(\mathrm{P})=1 \\
\text { si } \lambda_{1}<z<\lambda_{2} & \mathfrak{T}_{\lambda}(\mathrm{P})=\frac{1}{2}\left(1+\cos \theta_{1}\right), \quad \cos \theta_{1}=\frac{z}{\lambda_{1}} \\
\text { si } \lambda_{1}, \lambda_{2}<z & \mathfrak{T}_{\lambda}(\mathrm{P})=\frac{1}{2}\left(\cos \theta_{1}+\cos \theta_{2}\right), \cos \theta_{2}=\frac{z}{\lambda_{2}} .
\end{array}
$$

[Configuration of a rod near a wall. One monomer is fixed at $\mathbf{P}$, at a distance $z$ from the wall and at a distance $\lambda_{1}$ and $\lambda_{2}$ from the end of the rod.] dans le second cas (petite sphère de rayon $a, a<L$ ), les restrictions stériques sont moins importantes : la concentration est réduite essentiellement sur une distance de l'ordre de $a$. Qualitativement, les effets sont les mêmes qu'avec des polymères flexibles [11, 15], mais les profils de concentration sont différents et la déplétion en monomères moins forte car les effets entropiques sont plus faibles avec des polymères rigides qui ont des possibilités de configuration moins grandes que les polymères flexibles.

1.1.1 Profil de concentration près d'une paroi plane. - On cherche d'abord la probabilité $Q_{\lambda}(\mathrm{P})$ de trouver au point $P$ un monomère repéré par sa distance $\lambda$ de $\mathrm{P}$ à l'extrémité du bâton. $Q_{\lambda}(\mathrm{P})$ est proportionnelle au nombre de configuration accessible au bâton dans ce cas

$$
Q_{\lambda}(\mathrm{P})=1-\frac{\Omega_{\mathrm{int}}}{4 \pi}
$$

$\Omega_{\text {int }}$ est l'angle solide inaccessible au bâton à cause de la présence de la paroi avec le monomère $\lambda$ au point $\mathbf{P}$.

Nous résumons sur la figure 1 les différentes situations possibles et les probabilités associées. L'intégration sur tous les $\lambda$ possibles de 0 à $L$ donne le profil de concentration en monomère

$$
\begin{aligned}
c(z) & =c_{\mathrm{b}}\left(\frac{z}{L}\right)\left(1-\ln \frac{z}{L}\right) & \text { pour } z<L \\
& =c_{\mathrm{b}} & \text { pour } z>L
\end{aligned}
$$

Ce profil est quasi-linéaire, sauf aux extrémités de l'intervalle de variation. La déplétion est moins accentuée que dans le cas des polymères flexibles en solution semi-diluée et il sera peut-être plus difficile de l'observer directement, aussi bien par ellipsométrie que par d'autres méthodes plus récentes comme la fluorescence de chromophores sur la paroi excitée par les molécules au contact. Par contre, il existe un ordre orientationnel local, induit par la paroi, qui pourrait être plus facilement vu.

Comment évolue le profil de concentration quand la concentration globale augmente ? Les interactions entre bâtons favorisent l'alignement parallèlement à la paroi et le profil de déplétion s'adoucit sans doute davantage. Mais la longueur caractéristique reste d'ordre $L$, jusqu'à la transition nématique. En effet, on peut négliger pour les bâtons durs les effets prétransitionnels qui sont rencontrés dans les nématiques thermotropes [4]. La longueur de corrélation des orientations reste donc constamment d'ordre $L$ dans notre cas.

1.1.2 Profil de concentration en monomère près d'une sphère. - Le calcul se conduit de manière semblable à celui du paragraphe précédent (qui peut être inclu comme cas limite). Nous en présentons les éléments sur la figure 2. Si $a$ est le rayon de la sphère, et $r$ la distance au centre, nous trouvons dans le cas 
général, 3 expressions se prolongeant continuement (Fig. 3) :

- pour $0<r-a<\left(L^{2}+a^{2}\right)^{1 / 2}-a$

$$
\begin{aligned}
c_{\mathrm{I}} / c_{0}=\frac{1}{r}\left[\left(r^{2}-a^{2}\right)^{1 / 2}\right. & -\frac{a(r-a)}{2 L}+ \\
& \left.+\frac{1}{4}\left(\frac{r^{2}-a^{2}}{L}\right) \log \left(\frac{r-a}{r+a}\right)\right]
\end{aligned}
$$

- $\operatorname{pour}\left(L^{2}+a^{2}\right)^{2}-a<(r-a)<L$

$$
\begin{aligned}
c_{\mathrm{II}}(r) / c_{\mathrm{b}}=\frac{r-a}{L}-\frac{1}{4 r L}((r & \left.-a)^{2}-L^{2}\right)+ \\
& +\frac{1}{2 r L}\left(r^{2}-a^{2}\right) \log \frac{L}{r-a}
\end{aligned}
$$

- pour $r-a>L, c_{\mathrm{III}}(r)=c_{0}$.

On pose $x=\frac{r-a}{L}$. Voir figure 2.

$\mathrm{Si} a / L$ est très grand, on tombe sur le cas de la paroi plane

$$
c_{\mathrm{II}}(x) \simeq c_{\mathrm{b}} x(1-\log x)=c_{\mathrm{b}} \frac{r}{L}\left(1-\log \frac{r}{L}\right) .
$$

Nous nous intéressons au cas où $a / L$ est très petit devant l'unité, l'expression I est presque partout valable.

- A l'origine le comportement est singulier, pour $x L / a \ll 1$

$$
c_{\mathrm{I}}(r) / c_{\mathrm{b}} \sim\left(\frac{2 x L}{a}\right)^{1 / 2} .
$$

- Le comportement asymptotique pour

$$
\frac{a}{L} \ll x<1
$$

s'obtient en développant $c_{\mathrm{I}}$ en puissance de $(a / L)$

$$
c_{\mathrm{I}}(r) / c_{\mathrm{b}}=1-\frac{1}{2} \frac{(1-x)}{x^{2}}\left(\frac{a}{L}\right)^{2}+0\left(\frac{a}{L}\right)^{3} .
$$

Ces deux expressions montrent que la zone de déplétion s'étend sur une distance de l'ordre de $a$ et est très peu marquée. Il n'est pas question de l'observer directement, sauf éventuellement dans des expériences très lourdes de diffusion de neutrons. Nous verrons cependant qu'elle peut avoir des conséquences thermodynamiques très marquées (cf. 2).

1.2 Propriétés thermodynamiQues D'UNe SOLUTION CONFINÉE. - Nous décrivons les propriétés thermodynamiques dans le cas analytiquement simple d'une solution diluée confinée entre deux plaques.

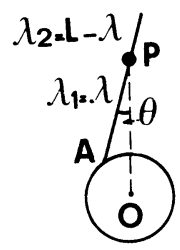

a1)

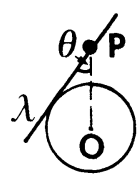

a2)

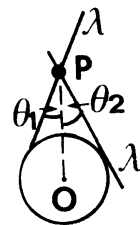

b) i)

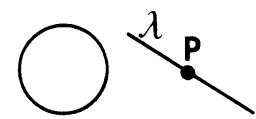

ii)

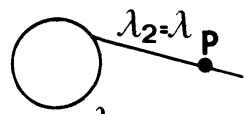

iii)

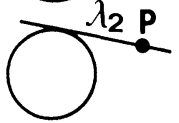

iv)

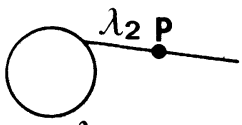

v)

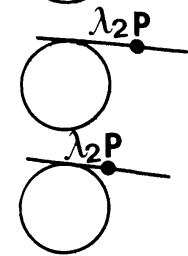

c)

Fig. 2. - a) Les différentes possibilités de contact entre un bâton de longueur $L$ et une sphère de rayon $a$.

$$
\begin{gathered}
\mathrm{AP}=\lambda, \quad \mathrm{OP}=r, \quad \mathrm{OA}=a, \\
\cos \theta=\frac{\lambda^{2}+r^{2}-a^{2}}{2 \lambda r} \quad \text { (figure gauche), } \\
\cos \theta=\frac{\left(r^{2}-a^{2}\right)^{1 / 2}}{r} \text { (figure droite) } .
\end{gathered}
$$

b) Angle solide accessible au bâton :

$$
\mathfrak{T}_{\lambda}(\mathrm{P})=\frac{1}{2}\left(\operatorname{Inf}\left(1, \cos \theta_{1}\right)+\operatorname{Inf}\left(1, \cos \theta_{2}\right)\right) .
$$

c) Les configurations respectives du bâton et de la sphère, quand un monomère est fixé en $P$ à une distance $r$ du centre de la sphère et une distance $\lambda$ de l'une des extrémités du bâton. Probabilités relatives associées.

i) $(r-a)>\lambda_{1}, \lambda_{2} \quad \mathfrak{T}_{\lambda}, 1=1$;

ii) $\left(r^{2}-a^{2}\right)^{1 / 2}>\lambda_{2}>(r-a)>\lambda_{1} \quad \mathfrak{T}_{\lambda}, 2=\frac{1}{2}\left(1+\frac{\lambda^{2}+r^{2}-a^{2}}{2 \lambda r}\right)$;

iii) $\lambda_{2}>\left(r^{2}-a^{2}\right)^{1 / 2}>(r-a)>\lambda_{1} \quad \mathfrak{T}_{\lambda}, 3=\frac{1}{2}\left(1+\frac{\left(r^{2}-a^{2}\right)^{1 / 2}}{2}\right)$;

iv) $\left(r^{2}-a^{2}\right)^{1 / 2}>\lambda_{2}>\lambda_{1}>(r-a)$

$$
\mathfrak{T}_{\lambda}, 4=\frac{1}{2}\left(\frac{\lambda^{2}+r^{2}-a^{2}}{2 \lambda r}+\frac{(L-\lambda)^{2}+r^{2}-a^{2}}{2(L-\lambda) r}\right)
$$

v) $\lambda_{2}>\left(r^{2}-a^{2}\right)^{1 / 2}>\lambda_{1}>(r-a)$

$$
\mathfrak{T}_{\lambda}, 5=\frac{1}{2}\left(\frac{\left(r^{2}-a^{2}\right)^{1 / 2}}{r}+\frac{\lambda^{2}+r^{2}-a^{2}}{2 \lambda r}\right) ;
$$

vi) $\lambda_{2}>\lambda_{1} \geqslant\left(r^{2}-a^{2}\right)^{1 / 2}>(r-a) \quad \mathfrak{j}_{\lambda}, 6=\frac{\left(r^{2}-a^{2}\right)^{1 / 2}}{r}$.

[a) The different possibilities of contact between a rod of length $L$ and a sphere of radius $a . b$ ) Solid angle accessible to the rod. $c$ ) The respective configurations of the rod and the sphere, when a monomer is fixed at $\mathbf{P}$ at a distance $r$ from the centre of the sphere and at a distance $\lambda$ from one end of the rod. Corresponding relative probabilities.]

On peut imaginer un film de solution, mais dans ce cas d'autres interactions entre les parois du film (électrostatique, dipolaire) compliquent la situation. 


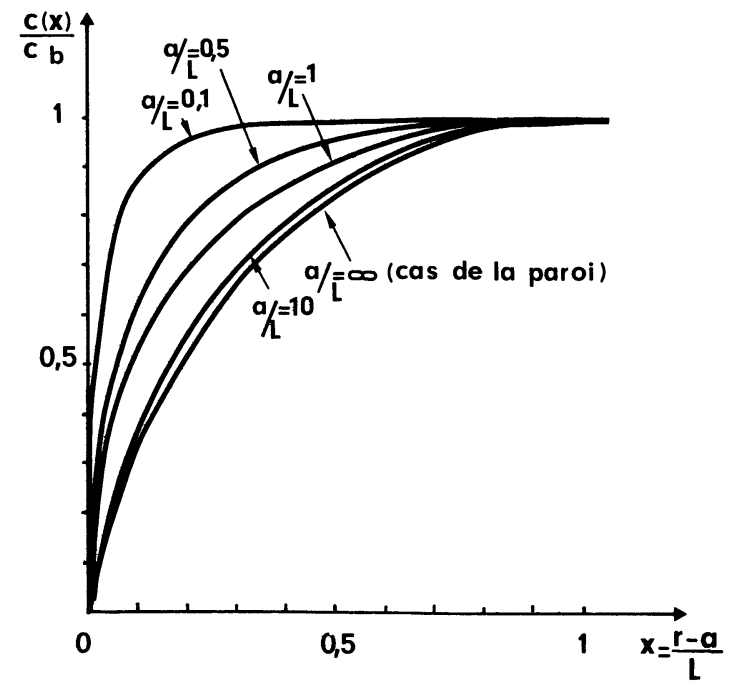

Fig. 3. - Profil de concentration en monomère près d'une sphère et d'un plan $a / L$ : rapport du rayon de la sphère à la longueur du bâton.

[Concentration profile of monomers near a sphere and a plane $a / L$ : ratio of sphere radius and rod length.]
Nous étendons la discussion à des géométries différentes et à des concentrations plus élevées.

1.2.1 Configuration d'un bâton entre deux plaques parallèles. Energie libre d'une solution. - L'existence de la zone de désorption près d'une paroi répulsive implique une énergie interfaciale positive que nous allons calculer.

Pour cela, il nous faut connaitre la fonction de distribution des centres de masse $\rho(z)$ et la fonction de distribution angulaire à centre de masse fixé $f(\theta / z)$ (normalisé à 1) $O z$ est l'axe perpendiculaire aux plaques, l'écartement entre les plaques est $D . \theta$ est l'angle d'un bâtonnet avec $\mathrm{Oz}$.

Posons $\Gamma=\int_{0}^{D} \rho(z) \mathrm{d} z, \Gamma$ est le nombre de bâtons confiné entre deux plaques de $1 \mathrm{~cm}^{2}$.

$\mathrm{Si} c_{\mathrm{p}}$ est la concentration moyenne en polymère (nb. de polymères par $\mathrm{cm}^{3}$ ) $\Gamma=c_{\mathrm{p}} D$.

Les mêmes considérations géométriques qu'au paragraphe précédent nous donne $\left(\theta_{0}\right.$ est défini par $\left.\cos \theta_{0}=2 z / L\right)$ :

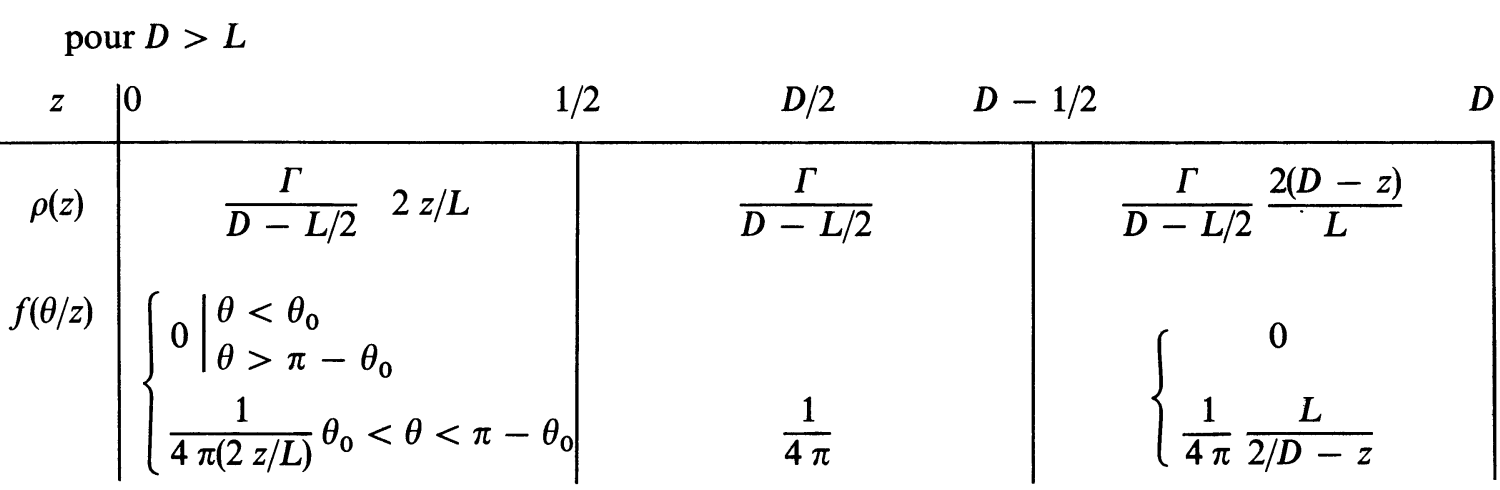

pour $D<L$

\begin{tabular}{|c|c|c|}
\hline$z$ & 0 & \\
\hline $\begin{array}{c}\rho(z) \\
f(\theta / z)\end{array}$ & $\left\{\begin{array}{l}\frac{2 P}{D}(2 z / D) \\
0 \begin{array}{l}\theta<\theta_{0} \\
\theta>\pi-\theta_{0} \\
L / 4 \pi(2 z)\end{array} \text { sinon }\end{array}\right.$ & $\begin{array}{l}2 P / D\left[\frac{2(D-z)}{D}\right] \\
\left\{\begin{array}{c}0 \\
L / 4 \pi 2(D-z)\end{array}\right.\end{array}$ \\
\hline
\end{tabular}

L'énergie libre par unité de surface est simplement d'origine entropique. Nous posons l'entropie rotationnelle nulle pour une distribution isotrope des orientations et l'entropie translationnelle nulle pour la concentration en polymère $\rho_{\mathrm{c}}$, d'empilement compact des bâtons.

L'énergie libre cherchée s'exprime donc par :

$$
F / T \mid \mathrm{cm}^{2}=\int_{0}^{D} \mathrm{~d} z 2 \pi \sin \theta \mathrm{d} \theta \rho(z) f(\theta / z) \log \left(4 \pi \frac{\rho(z)}{\rho_{\mathrm{c}}} f(\theta / z)\right) .
$$

Donc pour :

$$
\begin{array}{l|l}
D>L & F / T \mid \mathrm{cm}^{2}=\Gamma \log \frac{\Gamma}{D \rho_{\mathrm{c}}}-\Gamma \log (1-L / 2 D) \\
D<L / T \mid \mathrm{cm}^{2}=\Gamma \log \frac{\Gamma}{D \rho_{\mathrm{c}}}+\Gamma \log 2 L / D .
\end{array}
$$


Quand $D$ est très grand devant $L$, nous développons la première expression qui nous donne la contribution de l'énergie de surface de 2 parois : $2 \sigma$, où $\sigma$ est l'énergie interfaciale

$$
\sigma=\frac{1}{4} T L \frac{P}{D}=\frac{1}{4} T c_{\mathrm{p}} L
$$

Dans les deux cas, le premier terme de l'énergie libre est simplement le terme de pression d'une solution idéale de concentration $\Gamma / D$, le second représente la diminution d'entropie stérique et décrit une force supplémentaire $F$ repoussant les parois (à nombre de particules confinées constant ou $\Gamma$ constant). Dans le cas le plus intéressant $-D$ inférieur à $L-$ cette forme répulsive entre parois vaut, par unité de surface : $\Gamma T / D$.

On pourrait imaginer observer le confinement des bâtons dans un film liquide mince de solvant dont l'épaisseur serait, dans un modèle simplifié, uniquement stabilisée à cause de l'équilibre mécanique entre, d'une part, les forces de répulsion entropique dues aux bâtonnets et d'autre part, les forces d'attraction de Van der Waals qui s'exercent entre les interfaces (en l'absence de surfactants ioniques).

Malheureusement l'énergie libre $F_{\mathrm{s}}$ par unité de surface dont dérivent les forces (à $\Gamma$ fixé) :

$$
\left(F_{\mathrm{s}}=\Gamma T \log \frac{2 L}{D}-\frac{A}{12 \pi D^{2}}\right)
$$

n'a pas de minimum. L'équilibre n'est pas stable.

( $A$ est la constante de Hamaker [15] typiquement de l'ordre de $10^{-1} \mathrm{eV}$.)

Finalement nous pouvons évaluer le coefficient de partage entre une solution diluée de concentration $c_{\mathrm{po}}$ et une solution diluée confinée de concentration $c_{\mathrm{p}}$. Il vient :

$$
\begin{array}{ll}
\text { si } D>L & c_{\mathrm{p}}=c_{\mathrm{po}}\left(1-\frac{L}{2 D}\right) \\
\text { si } D<L & c_{\mathrm{p}}=c_{\mathrm{po}}\left(\frac{D}{2 L}\right) .
\end{array}
$$

1.2.2 Remarque sur le cas du confinement dans un pore. - Soit $D$ le diamètre du pore. Si $D$ est inférieur à $L$, le confinement et les restrictions stériques d'orientation d'un bâton est beaucoup plus fort que dans le cas d'une lame de même largeur que le pore. L'angle solide moyen dans lequel est confiné le bâton est de l'odre de $(D / L)^{2}$.

La loi d'échelle pour le coefficient de partage entre solutions diluées confinées de concentration $c_{\mathrm{p}}$ et libre de concentration $c_{\mathrm{po}}$ est alors :

$$
c_{\mathrm{p}} \sim c_{\mathrm{po}}\left(\frac{D}{L}\right)^{2}
$$

1.3 INTERACTIONS ENTRE SURFACES SOLIDES. Nous avons vu qu'il existait près des parois planes ou sphériques une zone de déplétion en monomère. Si nous considérons maintenant des particules colloïdales dures suspendues dans la solution de bâtonnet, nous voyons que dès qu'elles se trouvent à une distance l'une de l'autre inférieure à la longueur du bâton, leur zone de déplétion se recouvre et que l'énergie libre interfaciale de la solution sera minimisée si les particules se rapprochent. Cette situation est différente de celle évoquée précédemment, où on maintenait constant le nombre de particules confinées, ici, la quantité constante est le potentiel chimique des bâtons, autrement dit pour les solutions diluées, la concentration en molécules à l'infini.

Nous discutons successivement le cas de l'interaction en deux parois planes plongées dans la solution, puis l'interaction entre sphère de rayon $a(a \gg b$ et $a \ll L)$.

1.3.1 Interaction entre parois solides planes (Fig. 4). - Nous utilisons les résultats et les méthodes du paragraphe précédent pour calculer l'énergie libre de la solution enfermée dans le volume $\Omega=S X$ (cf. Fig. 4) contenant $\mathcal{N}=\Gamma S$ particules en présence et en l'absence des deux plaques espacées de $D$.

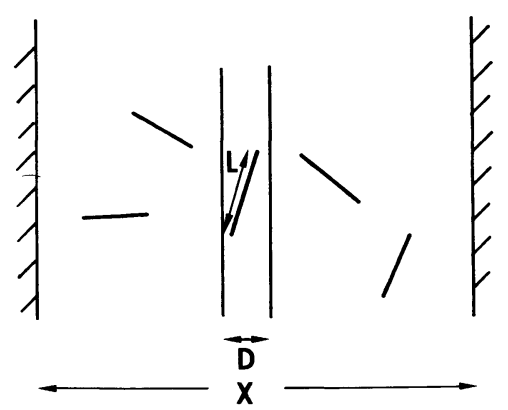

Fig. 4. - Deux parois solides dans une solution de bâton dur s'attirent dès que $D<L$. $D$ est l'écartement entre les parois, $S$ leur surface.

[Two solid walls in a hard rod solution attract each other for $D<L . D$ is the separation between walls, $S$ their surface areas.]

Nous trouvons en l'absence des plaques $(X \gg L)$ :

$F_{0} / T=\mathcal{N} \log \frac{\mathcal{N}}{S(X-L / 2) \rho_{\mathrm{c}}}=\mathcal{N} \log \frac{\mathcal{N}}{S X \rho_{\mathrm{c}}}+\frac{\mathcal{N}}{X} \frac{L}{2}$

(ce terme est l'énergie des parois en $\pm X / 2$ ) (cf. formule (7)) ;

en présence des plaques :

pour $\frac{D}{L}>1, \frac{F_{1}}{T}=\mathcal{N} \log \frac{\mathcal{N}}{S(X-3 L / 2) \rho_{\mathrm{c}}}$

$$
=\mathcal{N} \log \frac{\mathcal{N}}{S X \rho_{\mathrm{c}}}+\frac{\mathcal{N}}{X} \frac{3 L}{2}
$$


pour

$$
\begin{aligned}
& \frac{D}{L}<1, \frac{F_{1}}{T}= \\
& =\mathcal{N} \log \frac{\mathcal{N}}{S}\left[\left(X-L-D+\frac{1}{2} \frac{D^{2}}{L}\right) \rho_{\mathrm{c}}\right]^{-1} \\
& =\mathcal{N} \log \frac{\mathcal{N}}{S X \rho_{\mathrm{c}}}+\frac{\mathcal{N}}{X}\left[\left(L+D-\frac{1}{2} \frac{D^{2}}{L}\right)\right]^{-1} .
\end{aligned}
$$

A la limite thermodynamique :

$$
\begin{aligned}
& \mathcal{N} \rightarrow \infty \\
& X \rightarrow \infty \\
& X
\end{aligned}
$$

$c_{\mathrm{po}}$ est la concentration moyenne en bâtons.

L'énergie des deux plaques est (par unité de surface) :

$\frac{\Delta F}{T}=\frac{F_{1}-F_{0}}{T}=c_{\mathrm{po}} L$ si $\quad D>L$

$\frac{\Delta F}{T}=\frac{F_{1}-F_{0}}{T}=c_{\mathrm{po}}\left(\frac{L}{2}+D-\frac{1}{2} \frac{D^{2}}{L}\right)$ si $\quad D<L$.

Il existe une force attractive entre plaques pour $D<L$ qui vaut (par unité de surface) :

$$
\mathscr{F}=-\frac{\partial \Delta F}{\partial D}=-c_{\mathrm{po}}\left(1-\frac{D}{L}\right)
$$

1.3.2 Interaction entre sphères de grand rayon $(a \gg L) .-\mathrm{Si} l$ rayon de la sphère est très grand devant la longueur du bâton, nous pouvons utiliser l'expression (11) pour évaluer l'énergie d'interaction de deux sphères en sommant les contributions des surfaces en regard (cf. Fig. 5).

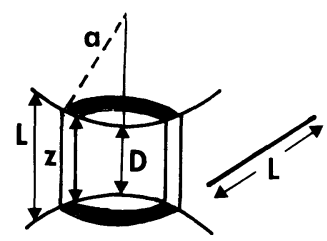

Fig. 5.- Deux sphères de grand rayon $(a \gg L)$ en interaction dans la solution de bâtonnet.

[Two spheres of large radius $(a \gg L)$ interacting in the hard rod solution.]

Si $z$ est la distance entre ces surfaces :

$$
\begin{aligned}
& \frac{\delta W_{\text {int }}}{T}=0 \text { si } \quad z>L, \\
& \frac{\delta W_{\text {int }}}{T}=c_{\mathrm{po}}\left(-\frac{L}{2}+z\left(1-\frac{z}{2 L}\right)\right) \text { si } \quad z<L .
\end{aligned}
$$

Soit $D+2 a$, la distance entre les centres des sphères $(D<L)$.

L'énergie d'interaction des deux sphères s'écrit alors :

$$
\begin{aligned}
\frac{W_{\text {int }}}{T}=\int_{0}^{L} \delta_{\text {int }}=(2 \pi a) \mathrm{d} z & =2 \pi a c_{\mathrm{po}}\left(-\frac{L}{2}(L-D)+\frac{1}{2}\left(L^{2}-D^{2}\right)-\frac{1}{6 L}\left(L^{3}-D^{3}\right)\right) \text { si } \quad D<L \\
& =0 \text { si } D>L
\end{aligned}
$$

et la force attractive induite entre les sphères vaut :

$$
\mathcal{F}=-\frac{\partial W}{\partial D}=2 \pi a c_{\mathrm{po}} T\left(D-\frac{D^{2}}{2 L}-\frac{L}{2}\right) \text { pour } D<L .
$$

Cette force est susceptible de déstabiliser la suspension de sphères dans la solution de bâtonnets si, pour $D=0, W_{\text {int }} / T$ est très grand devant $1-$ ce qui se produit à des concentrations relativement faibles pour $D=0$,

$$
\frac{W}{T}=-\frac{\pi}{z} c_{\mathrm{po}} a L^{2}
$$

et

$$
\frac{W}{T}(D=0) \gg 1 \quad \text { si } \quad c_{\mathrm{p}} \gg\left(L^{2} a\right)^{-1} \text {. }
$$

Nous verrons dans le paragraphe suivant qu'un effet semblable peut se produire avec des suspensions de petites sphères (pour des concentrations de bâtonnets un peu plus élevées).
1.3.3 Interaction entre petites sphères dans la solution. - Nous évaluons d'abord l'énergie d'une sphère dans la solution, puis l'énergie d'interaction de deux sphères, enfin nous discutons les conséquences possibles sur la déstabilisation d'une suspension de sphères.

a) Energie d'une sphère dans la solution. - $\mathrm{Si} a / L$ est très petit, les effets de surface sont prépondérants sur les effets de volume. Au premier ordre, l'augmentation d'énergie libre de la solution quand on y place la sphère est simplement le second terme du viriel de l'interaction entre un bâton et une sphère, en supposant la distribution de bâtons non perturbés.

Le volume exclu d'une sphère et d'un bâton de longueur $L$ et de direction $\mathbf{u}$ est simplement $\pi L a^{2}$ 
(cf. Fig. 6a). En moyennant sur u, cette valeur n'est pas changée et la self-énergie de la sphère dans la solution est :

$$
\frac{W}{T}=\pi c_{\mathrm{p}} L a^{2} \quad \text { si } \quad \frac{a}{L} \ll 1
$$

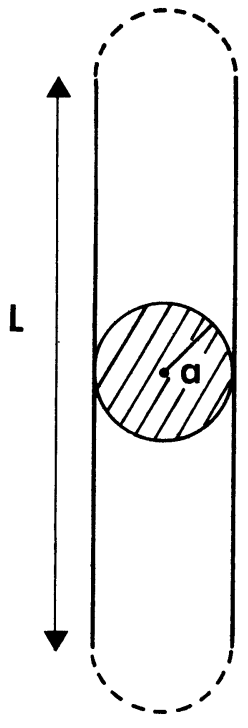

a)

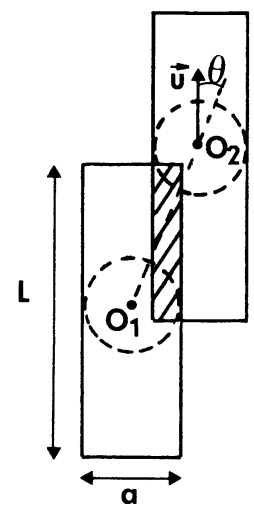

b)
Fig. 6. - a) Volume exclu d'un bâtonnet d'une sphère. b) Volume exclu d'un bâton orienté et de deux sphères. L'intersection des deux cylindres de longueur $L$ et de diamètre $2 a$ est représentée de profil.

(a) Excluded volume of a rod and a sphere. $b$ ) Excluded volume of an oriented rod and two spheres. Interaction of two cylinders of length $L$ and diameter $2 a$ represented in cross section.]

Le calcul plus précis de l'énergie libre de la solution à partir des fonctions de distribution des centres de masse et des orientations évalués à partir de raisonnements semblables à ceux du paragraphe précédent permet de retrouver ce résultat (cf. paragraphe 2.1.2 pour un calcul semblable sur le cas d'une paroi plane).
Introduisons la fraction volumique des bâtons $\varphi=c_{\mathrm{p}} L^{2} d$

$$
\frac{W_{\mathrm{sp}}}{T}=\pi \varphi\left(\frac{a}{d}\right)^{2}, \frac{W_{\mathrm{sp}}}{T}<1 \text { pour } a<d \varphi^{-1 / 2} .
$$

Si $a=(L d)^{1 / 2} W / T \sim 1$ à la transition nématique.

Négligeant les interactioons entre sphères, le coefficient de partage des sphères entre les phases isotropes et nématiques de la solution de bâtonnet dans la zone de coexistence est simplement donné par :

$$
\log \frac{\rho_{\mathrm{n}}}{\rho_{\mathrm{i}}}=-\pi a^{2} L\left(c_{\mathrm{pn}}-c_{\mathrm{pi}}\right)
$$

$\left(\rho_{\mathrm{i}}, \rho_{\mathrm{n}}\right.$ est la densité numérique des sphères dans la phase isotrope, resp. nématique). Si on prend $a$ tel que $W_{\mathrm{i}} / T=1$ comme à la transition nématique $c_{\mathrm{pn}} / c_{\mathrm{pi}}=2,34$ dans le modèle d'Onsager, on a

$$
\rho_{\mathrm{n}} / \rho_{\mathrm{i}}=\mathrm{e}^{-0,34} \sim 0,6
$$

la différence est donc très sensible.

b) Attraction entre petites sphères. - Si les zones de déplétion de deux sphères se recouvrent, celles-ci vont s'attirer en minimisant leur énergie libre de surface, $W_{2}$ que nous évaluons quand les deux sphères sont séparées par une distance $R$,

$$
\frac{W_{2}}{T}=c_{\mathrm{p}} B(R, a, L)
$$

où $B(R, a, L)$ est le volume exclu de l'haltère formé par les sphères et un bâton. Nous fixons l'orientation du bâton, u

$$
B(R, a, L, \mathbf{u})=2 \pi L a^{2}-V_{\text {int }}
$$

$V_{\text {int }}$ est le volume et l'intersection de 2 cylindres parallèles de rayon $a$, de longueur $L$, dont les centres sont distants de $R$ (cf. Fig. $6 b$ ).

$$
\begin{gathered}
V_{\text {int }}=0 \text { si } \frac{R \sin \theta}{2 a}>1 \\
R \cos \theta>L \quad \operatorname{sinon} \\
V_{\text {int }}=(L-R|\cos \theta|) a^{2}(\pi-2 \operatorname{arc}|\sin \theta|)-\sin 2\left(\arcsin \frac{R}{2 a}|\sin \theta|\right) .
\end{gathered}
$$

Si $a / L \ll 1$, posons

$$
\begin{aligned}
& \sin \theta_{0}=\theta_{0}=\frac{2 a}{R} \quad \text { si } \theta>\theta_{0} \quad V_{\text {int }}>=0 \\
& \theta<\theta_{0} \quad V_{\text {int }}<=(L-R) a^{2}\left(\pi-2 \arcsin \frac{\theta}{\theta_{0}}-2 \frac{\theta}{\theta_{0}}\left(1-\frac{1}{2} \frac{\theta}{\theta_{0}}\right)\right)
\end{aligned}
$$


Pour une distribution isotrope des bâtons

$$
\begin{aligned}
& \left\langle V_{\text {int }}\right\rangle=\int_{0}^{\theta_{0}} \mathrm{~d} \theta \theta V_{\text {int }}< \\
& B(R, L, a)=-L a^{2} \theta_{0}^{2} \int_{0}^{1} u \mathrm{~d} u\left(1-\frac{R}{L}\right)\left(\pi-2 \arcsin u-2 u\left(1-\frac{u}{2}\right)\right)+2 \pi L a^{2} \\
& =2 \pi L a^{2}-\left[\frac{\pi}{4}-\frac{5}{12}\right] L a^{2}\left(\frac{2 a}{R}\right)^{2}\left(1-\frac{R}{L}\right)+0\left(L a^{2}\left(\frac{a}{R}\right)\right)^{3} \\
& \frac{W_{2}}{c_{\mathrm{p}} T}=2 \pi L a^{2}-0,37 L a^{2}\left(\frac{2 a}{R}\right)^{2}\left(1-\frac{R}{L}\right) \text { pour } R \leqslant L \\
& =2 \pi L a^{2} \\
& a \ll L \\
& \text { pour } R \geqslant L \text {. }
\end{aligned}
$$

Ceci conduit à une force attractive entre sphères :

$$
\begin{array}{rlrl}
F=-\frac{\partial W_{2}}{\partial R} & =-0,19 T_{\mathrm{cp}} L a\left(\frac{2 a}{R}\right)^{3}\left(2-\frac{R}{L}\right) & \text { pour } & R \leqslant L \\
& =0 & \text { pour } \quad R \geqslant L .
\end{array}
$$

Pour deux sphères au contact : $R=2 a$

$$
\frac{W_{\text {int }}}{T}=0,37 c_{\mathrm{p}} L a^{2} \sim \varphi_{\mathrm{p}}\left(\frac{a}{d}\right)^{2}-\frac{W_{\text {int }}}{T}
$$

est de l'ordre de 1 si $\varphi_{\mathrm{p}}$ est de l'ordre de $d^{2} / a$ - comparons cette valeur à la fonction volumique à la transition nématique :

$$
\varphi_{\mathrm{n}}=\left(\frac{d}{L}\right)
$$

$\varphi_{\mathrm{p}} f$ sera inférieur à $\varphi_{\mathrm{n}}$ pour $a>(L d)^{1 / 2}$.

Cette interaction peut parfois être supérieure à l'interaction de Van der Waals entre deux sphères. $\mathrm{Si}$ les sphères sont proches

$$
V_{\mathrm{dw}}=\frac{A}{12 \pi} \frac{a}{R}[15] \text { pour } R=2 a
$$

$V_{\mathrm{dw}}=\frac{A}{24 \pi}$ si on prend $A$ de l'ordre de $10^{-1} \mathrm{eV}$, à température ordinaire $\frac{A}{T} \sim 4, \frac{V_{\mathrm{dw}}}{T} \sim 10^{-1}$.

Donc, dans le cas où les autres interactions sont faibles (interactions électrostatiques), on peut espérer induire uniquement avec les bâtonnets une flocculation entre sphères. Nous prévoyons aussi, qu'avec une solution nématique, on peut rendre le phénomène anisotrope (flocculation en filaments).

Remarque. - L'énergie d'interaction a le même comportement asymptotique que la concentration en monomères près d'une sphère. Cela tient au fait qu'un second ordre en l'interaction, l'énergie d'une sphère dans le champ de déplétion de l'autre est simplement :

$$
W(R) / T=c_{\mathrm{p}} \pi L a^{2} g(R)
$$

où $g(R)$ est la fonction de corrélation de paire monomère-sphère. $g(R)$ n'est rien d'autre que $c(R) / c_{\mathrm{b}}$ et $c_{\mathrm{p}} \pi L a^{2}$ l'intensité de l'interaction bâton-sphère.

L'énergie de deux sphères est alors :

$$
\begin{gathered}
W / T=c_{\mathrm{p}} \pi L a^{2}(1+g(R))= \\
=2 c_{\mathrm{p}} \pi L a^{2}-\pi / 8\left(\frac{2 a}{R}\right)^{2}\left(1-\frac{R}{L}\right) \\
\pi / 8=0,39 .
\end{gathered}
$$

Les deux méthodes de calcul sont donc en bon accord (à $5 \%$ près).

\section{Quelques aspects dynamiques du problème. -} Peut-on émettre des idées sur le comportement rhéologique de ces solutions de bâtonnets en milieu confiné quand les effets à l'équilibre sont si mal connus? Pour les solutions diluées, oui. Ces solutions sont d'ailleurs plus simples à étudier en pratique, car moins visqueuses que des solutions concentrées.

L'essentiel de la discussion portera donc sur des solutions diluées (dans un pore de diamètre $D$, la concentration en polymères $c_{\mathrm{p}}$ est telle que $\left.c_{\mathrm{p}} L D^{2} \ll 1\right)$. Nous ferons quelques conjectures sur le cas semi-dilué dans l'esprit de Doi et Edwards. Enfin, nous discuterons aussi la viscosité $\eta(q)$ d'une solution de bâtons lorsque le gradient de vitesse est modulé spatialement avec une longueur d'onde $2 \pi / q$. Nous indiquerons quelques cas pratiques où la connaissance de $\eta(q)$ peut être utile. 
2.1 RÉDUCTION DE VISCOSITÉ APPARENTE EN MILIEU CONFINÉ. - En vue d'applications en récupération -assistée du pétrole, G. Chauveteau [10] a étudié le comportement rhéologique d'une solution de polysaccharide de type xanthane, dans des matériaux poreux (sableux) et dans des nucléopores. Dans ces pores de très petites dimensions, la réduction de la viscosité par rapport à sa valeur en milieu non confiné est très nette, aussi bien en régime newtonien que non newtonien. Il l'attribue à la déplétion en polymère près de la paroi et l'interprète à l'aide d'un modèle rhéologique à deux viscosités : une pour le centre du pore, une pour la zone limite. Les deux paramètres (largeur de la zone limite, viscosité dans cette zone) interprètent bien les résultats expérimentaux. La relation entre la largeur de la zone limite, la longueur de la molécule et le taux de cisaillement est cependant encore peu précise, d'autant plus qu'il faudrait prendre en compte une légère flexibilité des bâtonnets.

Nous présentons ci-dessous l'étude d'un cas simple qui intègre les effets de déplétion.

a) Ecoulement de cisaillement entre deux plaques. Une des plaques se meut par rapport à l'autre et crée en les plaques un écoulement de cisaillement transversal. Le taux de cisaillement est $\dot{\gamma}$, l'écartement des plaques $D(D<L)$.

Nous calculons la viscosité en évaluant la dissipa- tion moyenne par bâton $T \dot{S}$. Nous négligeons les interactions hydrodynamiques molécule-paroi et monomère-monomère (modèle de Rouse).

Kirkwood et Auer [6] ont montré que la prise en compte des interactions hydrodynamiques entre monomères d'un bâtonnet n'introduisait que des connections logarithmiques dont nous ne tiendrons pas compte dans notre modèle simple.

La dissipation d'énergie par bâton s'écrit :

$$
T \dot{S}=\int_{0}^{D} \mathrm{~d} z \mathrm{~d} \Omega P(z) f(\Omega / Z) \sum_{i=-N / 2}^{N / 2} \mathbf{F}_{i} \cdot \mathbf{v}_{i}
$$

$P(z) f(\Omega / z)$ est la probabilité pour un bâton d'avoir son centre de masse en $z$, sur l'axe perpendiculaire aux plaques et une orientation $\Omega . v_{i}$ est la vitesse du fluide au point où se trouve $l$ monomère $i . v_{i}=\dot{\gamma} z_{i}$. $F_{i}$ est la force de friction exercée par le fluide sur le monomère $i$.

Le coefficient de friction d'un monomère est $\zeta\left(\zeta \sim \eta_{\mathrm{s}} L / N\right.$ où $\eta_{\mathrm{s}}$ est la viscosité du solvant).

A l'ordre zéro, dans le domaine newtonien, la distribution des positions et des orientations n'est pas perturbée par l'écoulement. $P(z)$ et $f(\Omega / z)$ sont donnés par les formules (6).

Donc :

$$
\begin{aligned}
& T \dot{S}=\int_{0}^{D} \mathrm{~d} z \mathrm{~d} \Omega P(z) f(\Omega / z) \zeta \dot{\gamma}^{2} \sum_{i=-N / 2}^{N / 2} \frac{L^{2}}{N} i^{2} \cos ^{2} \theta=\frac{N}{z} \zeta L^{2} \dot{\gamma}^{2} \int_{0}^{D} \mathrm{~d} z \mathrm{~d} \Omega P(z) f(\Omega / z) \cos ^{2} \theta \\
& \int_{0}^{D} \mathrm{~d} z P(z) f(\Omega / z)=2 \int_{0}^{D / 2} \mathrm{~d} z \frac{2}{D}\left(\frac{2 z}{D}\right) \int_{\theta_{0}}^{\pi-\theta_{0}} 2 \pi \sin \theta \mathrm{d} \theta \frac{L}{4 \pi(2 z)} \cos ^{2} \theta\left(\cos \theta_{0}=2 z / L\right) .
\end{aligned}
$$

Il vient

$$
T \dot{S}=\frac{1}{18} N \zeta L^{2}\left(\frac{D}{L}\right)^{2} \dot{\gamma}^{2}
$$

Si $\Gamma$ est le nombre de bâtons par unité de surface, la dissipation par unité de surface vaut $\frac{1}{18} \Gamma N \zeta D^{2} \dot{\gamma}^{2}$, qu'on peut écrire $\delta \eta \dot{\gamma}^{2} D . \delta \eta$ est l'accroissement de viscosité dû au polymère

$$
\delta \eta=\frac{1}{18} \frac{\Gamma}{D} N \zeta D^{2}=\frac{1}{18} c \zeta D^{2}
$$

où $c$ est la concentration moyenne en monomères dans le tube.

Un raisonnement simple permet de retrouver la forme de ce résultat et de la généraliser.

b) Lois d'échelle. - L'accroissement de viscosité d'une solution polymérique se met souvent sous la forme $[E][\tau][17]$ où $E$ est un module d'élasticité, en général peu variable $E=c T / N$. $\tau$ est un temps càractéristique de la molécule, on peut le concevoir comme le temps mis par la macromolécule à explorer ses configurations quand elle dissipe à cause de l'écoulement.

Ici, ce temps caractéristique est le temps de rotation brownienne des bâtons dans l'angle solide d'ouverture $D / L$. Si $\tau_{0}^{-1}$ est le coefficient de rotation du bâton libre

$$
\left(\frac{D}{L}\right)^{2}=\tau_{0}^{-1} \tau \quad \text { avec } \quad \tau_{0} \sim \eta_{\mathrm{s}} L^{3} / T \sim \zeta \frac{N}{T} L^{2} .
$$

Donc

$$
\delta \eta=\langle c\rangle \zeta L^{2}\left(\frac{D}{L}\right)^{2}=\langle c\rangle \zeta D^{2}
$$

où $c$ est la concentration moyenne dans le tube. $\delta \eta$ est indépendant de $L$ pour $D<L$. 
Le même raisonnement d'échelle peut s'appliquer pour un écoulement de Poiseuille dans une lame ou un pore, il est probable que dans ce cas, la dépendance en $D$ de la viscosité soit la même. Ce résultat pourrait être vérifié dans des nucléopores de longueur suffisante pour que les "effets d'entrée " soient négligeables. (Pour une discussion des effets d'entrée, voir section 3.)

c) Effets d'enchevêtrement en solution semi-diluée, dans un tube. - Si $c_{\mathrm{p}}$ est la concentration en bâtonnets, dans un tube de diamètre $D$, les effets d'enchevêtrement vont apparaître pour $c_{\mathrm{p}}>c_{\mathrm{p}}^{*}\left(c_{\mathrm{p}}^{*} L D^{2} \sim 1\right)$. (Les molécules sont d'ailleurs moins enchevêtrées à cause de leur orientation par le tube.) Le temps de rotation d'une molécule est considérablement allongé et est dominé par le temps de résorption d'un enchevêtrement.

Nous négligeons les effets de déplétion et nous supposons que la distribution des orientations est uniforme dans un cône d'ouverture $D / L$ d'axe parallèle à celui du tube.

Dans l'esprit de la reptation, D.E. introduisent autour d'une molécule un cylindre de diamètre $a_{\mathrm{c}}$ tel que le nombre de bâtons qui touchent le cylindre soit en moyenne $1=N\left(a_{\mathrm{c}}\right)$ [cf. Fig. (7)].

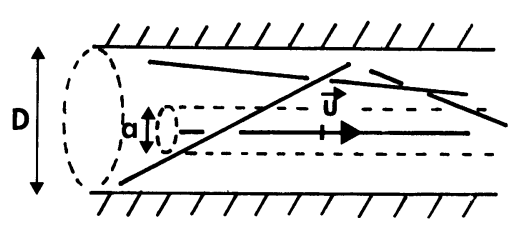

Fig. 7. - Bâtonnets enchevêtrés dans un pore. On évalue le nombre de molécules intersectant le cylindre de diamètre $a$, de longueur $L$.

[Entangled rods in a pore, we evaluate the number of molecules intersecting the cylinder of diameter $a$ and length $L$.]

Pour une distribution d'orientation $\mathbf{u}, f(\mathbf{u})$

$$
N(a, \mathbf{u})=2 c_{\mathrm{p}} a L^{2} \int \mathrm{d}^{2} \mathbf{u} \sin \left(u u^{\prime}\right) f\left(u^{\prime}\right) .
$$

Pour des angles petits (confinement fort) :

$$
\begin{aligned}
\sin \left(\mathbf{u}, \mathbf{u}^{\prime}\right) & =\left(\theta^{2}+\theta^{\prime 2}-2 \theta \theta^{\prime} \cos \varphi^{\prime}\right)^{1 / 2} ; \\
f(\theta) & =\frac{1}{\pi(D / L)^{2}} \quad \text { si } \quad \theta<D / L ;
\end{aligned}
$$

alors :

$$
\begin{aligned}
N(a, \mathbf{u})=\frac{2 c a L^{2}}{\pi} & \frac{D}{L} \int_{0}^{2} \mathrm{~d} \varphi^{\prime} \times \\
& \times \int_{0}^{1} y \mathrm{~d} y\left(x^{2}+y^{2}-2 x y \cos \varphi^{\prime}\right)^{1 / 2}
\end{aligned}
$$

sinon :

$$
x=\theta /(D / L), \quad y=\theta^{\prime} /(D / L)
$$

en moyennant sur $\mathbf{u}$ :

$$
\langle N(a)\rangle \sim c_{\mathrm{p}} a L D \text { on en déduit } a_{\mathrm{c}} \sim 1 / c_{\mathrm{p}} L D,
$$

on vérifie bien que $a_{\mathrm{c}} \ll D$ pour $c_{\mathrm{p}} L D^{2} \gg 1$.

Pour $c_{\mathrm{p}}=c_{\mathrm{p}}^{*} \sim 1 / L D^{2}, a_{\mathrm{c}}=D$. A cette concentration, le temps $\tau_{\mathrm{D}}$ de rotation du bâton dans l'angle solide $(D / L)^{2}$ est celui d'un bâton en solution diluée :

$$
c_{0}=c_{0 \mathrm{D}}=\eta_{\mathrm{s}} \frac{L^{3}}{T}\left(\frac{D}{L}\right)^{2} .
$$

En moyenne, $a_{\mathrm{c}} / L$ est le pas élémentaire de la diffusion rotatoire du bâton en solution semi-diluée enchevêtrée. Dans cette situation, la loi d'échelle de la diffusion impose que :

$$
\frac{\tau_{0 \mathrm{D}}}{\tau_{\mathrm{D}}}=\left(\frac{a_{\mathrm{c}}}{D}\right)^{2} .
$$

Pour libérer un enchevêtrement, il faut qu'une molécule se translate le long de son axe et tourne, car en général la paroi empêche la translation dans une direction quelconque, $\tau_{\mathrm{DD}}$ est en moyenne le temps de contournement et de désenchevêtrement.

Finalement

$\tau_{\mathrm{D}}=\tau_{0 \mathrm{D}}\left(c L D^{3}\right)^{2}=\tau_{0 \mathrm{D}}\left(\frac{c_{\mathrm{p}}}{c_{\mathrm{p}}^{*}}\right)^{2}=\tau_{\text {D.E. bulk }}\left(\frac{D}{L}\right)^{6}$.

A concentration égale, ce temps est donc considérablement réduit par rapport à la valeur bulk.

En conclusion, il faut cependant souligner le caractère très conjonctural de l'expression (17), due principalement au traitement grossier des effets de surface et de confinement sur la dynamique du bâton.

2.2 Viscosité AUX PETITes ÉCHelles D'UNe SOLUTION DE BÂTONNET. - Le mouvement d'une petite sphère comme celle du paragraphe (1.3.3) crée dans la solution de bâtonnet une perturbation d'écoulement, donc une dissipation qui ne fait pas intervenir la viscosité macroscopique de la solution. La sphère sonde des détails à une échelle inférieure à $L$ et perturbe peu le milieu. Ceci peut constituer (pour certains cas plus complexes), une méthode originale de tester la dynamique et les corrélations locales de la solution, applicable aussi aux polymères flexibles [8]. On a pu mesurer ainsi la longueur de corrélation d'une solution diluée de flexibles [17].

Il serait très lourd de calculer exactement les interactions hydrodynamiques de la sphère et des bâtons dans la solution. Si le rayon de la sphère est $a$, nous pouvons définir la viscosité vue par la sphère $\eta_{\text {app }}$ par la loi de Stokes. Si $\mu$ est la mobilité de la solution, nous posons $\eta_{\text {app }}=\mu / 6 \pi a$, ce qui définit $\eta_{\text {app }}$ pratiquement. Théoriquement, nous supposons qu'elle suivra la même loi d'échelle que celle calculée pour une perturbation d'écoulement modulée spatialement à la longueur d'onde $2 \pi / q=a$. Nous verrons d'ailleurs 
que cette loi est la même que celle du fluide confiné dans une lame d'épaisseur $D=2 \pi / q^{\prime}$.

Nous allons donc étudier la friction subite par un

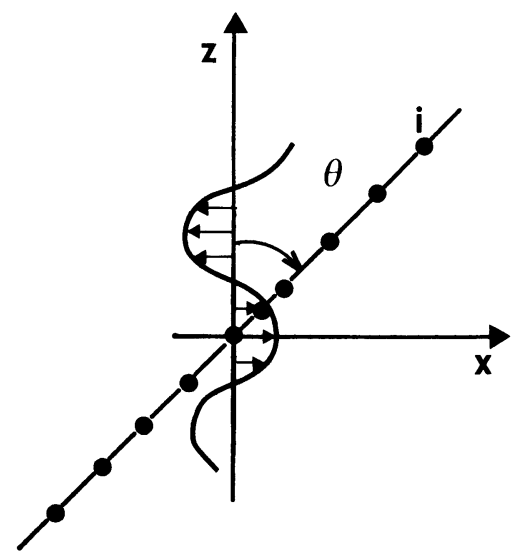

Fig. 8. - Bâton dans un écoulement de cisaillement longitudinal. [Rod in longitudinal shear flow.] bâton dans un tel écoulement. Le champ des vitesses est $v_{x}=v_{0} \cos q z, v_{y}=0, v_{z}=0$ (cf. Fig. 8). Comme aux paragraphes précédents, nous négligeons les interactions hydrodynamiques. Le coefficient de friction d'un monomère est $\zeta$.

Pour de grands vecteurs d'ondes $(q L \gg 1)$ et des vitesses petites, l'écoulement n'a pas d'action motrice sur le bâton, et celui-ci garde une répartition isotrope de ses orientations. Ce qu'il ressent ne dépend pas, à une petite modulation près, de la position de son centre de masse. La dissipation due à un bâtonnet dont le centre de masse est à l'origine et l'axe fait un angle $\theta$ avec $O z$ est :

$$
\begin{aligned}
T \dot{S}(\theta) & =\zeta \sum_{i=-N / 2}^{N / L} v_{0}^{2} \cos ^{2} z_{i}, \quad z_{i}=\frac{L}{N} i \cos \theta \\
& =\zeta v_{0}^{2} N / 21+\frac{\sin q L \cos \theta}{q L \cos \theta}
\end{aligned}
$$

par unité de volume, la dissipation moyenne est donc :

$$
\begin{aligned}
& T \dot{S}=\frac{c}{N} T\langle\dot{S}(\theta)\rangle=\frac{1}{2} c \zeta v_{0}^{2}\left[1+\frac{1}{2} \int_{0}^{D} \mathrm{~d} \theta \sin \theta \frac{\sin q L \cos \theta}{q L \cos \theta}\right] \\
& T \dot{S}=\frac{1}{2} c \zeta v_{0}^{2}\left[1+\frac{1}{2} \int_{-1}^{1} \mathrm{~d} u \frac{\sin q L n}{q L n}\right]=\frac{c \zeta v_{0}^{2}}{2}\left[1+\frac{1}{q L} \int_{0}^{q L} \mathrm{~d} y j_{0}(y)\right],
\end{aligned}
$$

pour $q L \gg 1$ :

$$
T \dot{S}=\frac{c \zeta c_{0}^{2}}{2}=\frac{1}{2} \frac{c \zeta}{q^{2}} \dot{\gamma}
$$

$\dot{\gamma}$ est le gradient de cisaillement.

Par définition, $T \dot{S}=\delta \eta(q) \dot{\gamma}^{2}$. Donc pour $q L \gg 1$ :

$$
\delta \eta(q)=\frac{1}{2} c \zeta q^{-2} .
$$

Ce résultat peut être retrouvé par un argument d'échelle. L'accroissement de viscosité newtonien à gradient uniforme est $: \delta \eta(0) \sim c \zeta L^{2}$, on postule

$$
\delta \eta(q) \sim c \zeta L^{2} f(q L), \quad f(x)=1, \quad x \rightarrow 0
$$

pour de grands vecteurs d'onde, la viscosité ne doit plus dépendre de $L$. L'écoulement est "myope». Ceci impose la dépendance $\delta \eta(q) \sim q^{-2}$.

Ainsi, la viscosité ressentie par une bille de rayon $a$ dans la solution sera typiquement :

$$
\begin{array}{rlr}
\eta & =\eta_{\mathrm{s}}+\alpha c \zeta a^{2} & d=0(1) \\
& =\eta_{\mathrm{s}}+\beta c_{\mathrm{p}} \eta_{\mathrm{s}} L a^{2} & \beta=O(1) \\
\eta & =\eta_{\mathrm{s}}\left(1+\beta c_{\mathrm{p}} L a^{2}\right), &
\end{array}
$$

$c$ est la concentration en monomères au voisinage de la bille. On peut négliger dans ce cas les effets de déplétion. $c$ est identique à la concentration globale.
En faisant sédimenter la bille sans perturber la solution de bâtonnet (ce qui est possible car les échelles de temps de la bille et d'un bâton sont très différentes), ou en étudiant la diffusion de la bille, on peut vérifier cette loi qui n'est rien d'autre que l'estimation d'un coefficient de Huggins bille-bâton.

\section{Effets d'écoulements à l'entrée d'un capillaire} fin. - Quand on applique une différence de pression de part et d'autre d'une membrane poreuse, il se crée des écoulements convergents à l'entrée des pores. Si les pores sont suffisamment épais, on peut étudier l'action de l'écoulement dû à un pore unique sur la solution (Fig. 10). Dans ce cas, il existe en chaque point loin de la paroi et en dehors de la zone d'entrée un gradient de vitesse longitudinal dirigé vers l'entrée du pore. Pour étudier l'effet de cet écoulement, sur des solutions diluées et semi-diluées, nous procédons en deux étapes :

i) nous étudions l'action d'un écoulement de cisaillement longitudinal uniforme sur la solution. Nous étendons phénoménologiquement l'analyse de Kramers [5] au cas des solutions semi-diluées (en intégrant l'effet d'enchevêtrement et des interactions de volume exclu). Cette section dépasse dans ses applications le cadre de l'article, mais permet d'aborder le cas plus complexe de l'écoulement convergent; 
ii) nous décrivons le comportement de la solution dans l'écoulement convergent vers l'entrée du capillaire et les effets d'entrée (orientation, passage, relaxation) selon la valeur du flux de solvant.

3.1 SOLUTIONS DE BÂTONS DURS SEMI-DILUÉS DANS UN ÉCOULEMENT DE CISAILLEMENT TRANSVERSAL HOMOGÈNE. - L'étude que nous entreprenons ici n'a pour but que de décrire l'allure générale des phénomènes et de donner des ordres de grandeur qui nous guiderons dans la section suivante. La dynamique et la rhéologie des solutions semi-diluées sont très complexes et nous n'espérons pas atteindre la rigueur des résultats que donne la résolution d'une équation de convectiondiffusion, comme celle de Doi et Edwards [7].

La difficulté vient des effets de volume exclu, qui se manifestent dans la région semi-diluée $\left(c^{*}<c<c_{\mathbf{n}}^{*}\right)$ de deux manières très différentes pour des bâtons très longs :

i) ils favorisent l'alignement [1];

ii) la rotation des macromolécules est ralentie à cause des enchevêtrements entre bâtons, d'autant plus que les molécules sont moins alignées [7].

Ces circonstances introduisent des effets non linéaires dont une théorie dynamique de champ moyen comme celle récemment proposée par Hess [18] pour décrire la transition nématique sans écoulement de cisaillement transversal, ne peut rendre compte.

En régime permanent, nous décrivons une théorie semi-phénoménologique qui reprend certaines idées de la théorie du champ moyen et devrait permettre de tenir compte de tous les effets de l'interaction stérique entre particules. Nous donnons la variation $\mathrm{du}$ paramètre d'ordre en fonction du gradient de vitesse et de la concentration. Essentiellement, loin de la transition nématique, les gradients de vitesse nécessaires à l'orientation sont plus faibles qu'en régime dilué - la solution devient rapidement non newtonienne - et les effets non linéaires n'ont un rôle dramatique que près de la transition nématique, ou pour des gradients très forts.

L'hypothèse de régime permanent n'est qu'une idéalisation dans beaucoup de situations expérimentales. Dans un écoulement convergent à l'entrée d'un capillaire ou dans l'expérience de siphon sans tube, la molécule entraînée par le fluide voit varier le taux de cisaillement transversal et ne passe qu'un temps fini dans la zone où celui-ci existe effectivement. Pour ces régimes non stationnaires, nous proposons une équation de relaxation applicable loin de la transition nématique.

3.1.1 Distribution des orientations dans une hypothèse de régime permanent. - a) En solution très diluée, on sait depuis Kramers [5] que la distribution des orientations d'un bâtonnet dans un gradient de vitesse d'écoulement longitudinal peut être décrite par une énergie potentielle effective :

$$
U=-T\left(\tau_{0} \dot{\gamma}\right) P_{2}(\theta),
$$

$\dot{\gamma}$ est le gradient de cisaillement de l'écoulement,

$$
\begin{gathered}
V_{x}=-\frac{1}{2} \dot{\gamma} x ; \quad V_{y}=-\frac{1}{2} \dot{\gamma} y ; \quad V_{z}=\dot{\gamma} z ; \\
P_{2}(\theta)=\frac{1}{2}\left(3 \cos ^{2} \theta-1\right),
\end{gathered}
$$

$\theta$ est l'angle de l'axe d'une molécule avec $\mathrm{O} z$, $\tau_{0} \sim \eta_{\mathrm{s}} L^{3} / T, T$ est la température (en unité d'énergie).

Et la fonction de distribution des orientations d'un bâton est :

$$
f(\theta)=\frac{1}{Z} \exp \left(\tau_{0} \dot{\gamma}\right) P_{2}(\theta) .
$$

b) Quand la solution est moins diluée et que les interactions entre bâtonnets deviennent importantes, nous postulons que la fonction de distribution garde la forme de Maier-Saupe :

$$
f(\theta)=\frac{1}{Z} \exp \left[g\left(S, c_{\mathrm{p}}, \dot{\gamma}\right) P_{2}(\cos \theta)\right]
$$

avec

$$
S=\left\langle P_{2}(\theta)\right\rangle
$$

$S$ mesure l'ordre orientationnel de la solution.

C'est une des formes les plus simples compatibles avec la symétrie du problème et la forme de la distribution dans les cas limites : écoulement d'une solution diluée ou solution au repos [4].

$g\left(S, c_{\mathrm{p}}, \dot{\gamma}\right)$ inclut de manière "self consistente " les effets de volume exclu et d'orientation par l'écoulement.

Nous développons $g$ en fonction de $\dot{\gamma}$ et de $S$. $P_{2}(\cos \theta)$ et $\dot{\gamma}$ sont les éléments d'un tenseur et l'argument de l'exponentielle un scalaire. Ceci implique que $g$ soit aussi l'élément d'un tenseur et impose les symétries du développement de $g$ en fonction de $S$ et $\dot{\gamma}$. Pour $S=0, \dot{\gamma}=0$ la distribution est isotrope. Donc, si on écrit :

$$
g(S, \dot{\gamma})=a(\dot{\gamma})+b(\dot{\gamma}) S+c(\dot{\gamma}) S^{2}
$$

au premier ordre en $\dot{\gamma}$ :

$$
a(\dot{\gamma})=\tau \dot{\gamma} ; \quad b(\dot{\gamma})=\lambda ; \quad c(\dot{\gamma})=\lambda^{\prime}+\tau^{\prime} \dot{\gamma} ;
$$

(nous négligerons les termes d'ordre supérieur).

* $\lambda$ et $\lambda^{\prime}$ rendent compte des effets de volume exclu.

Dans l'approximation de champ moyen, à symétrie quadrupolaire (Maier-Saupe), on prend

$$
\begin{aligned}
\lambda & =4,5 c_{\mathrm{p}} / c_{\mathrm{pn}} \\
\lambda^{\prime} & =0
\end{aligned}
$$

$c_{\mathrm{pn}}$ est la concentration de transition nématique.

A gradient nul, on retrouve la distribution de Maier-Saupe pour des bâtons. 
* $\tau$ et $\tau^{\prime}$ rendent compte des effets d'orientation par le gradient et des effets d'enchevêtrement.

- En solution diluée :

$$
\begin{aligned}
\tau & =\tau_{0} \sim \eta_{\mathrm{s}} L^{3} / T \\
\tau^{\prime} & =0 .
\end{aligned}
$$

- En solution semi-diluée : $\tau$ et $\tau^{\prime}$ seront déterminés ici au moyen de l'analyse de Doi et Edwards.
Leur temps de rotation d'un bâton dans la solution isotrope est $\tau_{\mathrm{r}} \sim \eta_{\mathrm{s}} L^{3} / T\left(c L^{3}\right)^{2}$. Cependant, comme nous l'avons déjà vu, quand les molécules sont polarisées, les enchevêtrements jouent un rôle moins important et la diffusion rotationnelle d'un bâton est plus facile et dépend de l'orientation et de la fonction de distribution. Doi et Edwards écrivent un coefficient de diffusion pour ce cas :

$$
D_{\mathrm{r}}[f, \mathbf{u}]=\frac{1}{\tau_{\mathrm{r}}}\left[4 / \pi \int \mathrm{d}^{2} u^{\prime} f\left(\mathbf{u}^{\prime}\right)\left|\sin \left(\mathbf{u}, \mathbf{u}^{\prime}\right)\right|\right]^{-2} .
$$

Nous l'évaluons à l'approximation quadrupolane :

$$
\begin{gathered}
\int f\left(\mathbf{u}^{\prime}\right) \sin \left|\mathbf{u}, \mathbf{u}^{\prime}\right| \mathrm{d}^{2} u^{\prime}=\frac{\pi}{4}-\frac{5 \pi}{2^{5}}\langle S\rangle P_{2}(\cos \theta) \quad\langle S\rangle=\left\langle P_{2}(\cos \theta)\right\rangle \\
\left(\int \mathrm{d}^{2} \mathbf{u}^{\prime} f\left(\mathbf{u}^{\prime}\right)\left|\sin \left(\mathbf{u}, \mathbf{u}^{\prime}\right)\right|\right)^{2}=\left(\frac{\pi}{4}\right)^{2}\left(1-\frac{5}{2^{3}} S P_{2}(\cos \theta)\right)^{2} \sim\left(\frac{\pi}{4}\right)^{2}\left(1-\frac{5}{4} S P_{2}(\cos \theta)\right) .
\end{gathered}
$$

En moyennant, il apparaît un temps $\tau_{\mathrm{r}}(S)=1 / D_{\mathrm{r}}(S)$ qu'on interpole par $\tau_{\mathrm{r}}(S)=\tau_{\mathrm{r} \text { isotrope }}\left(1-S^{2}\right)$.

Les effets de gradient sont les suivants :

- quand les molécules sont peu orientées et fortement emmêlées, la diffusion rotationnelle est fortement ralentie : l'écoulement peut orienter plus facilement les molécules;

- quand l'orientation est forte, il peut arriver, à concentration relativement faible, qu'on désenchevêtre complètement les molécules. Les molécules regagnent alors leur statut de particules libres et recouvrent leur temps caractéristique $\tau_{0}$.

On choisit donc d'écrire la constante de couplage orientationnel comme :

$$
\left(\tau+\tau^{\prime}\right) \dot{\gamma}=\left[\tau_{0}+\tau_{\mathrm{r}}\left(1-S^{2}\right)\right] \dot{\gamma} \quad \tau_{0} \sim \eta_{\mathrm{s}} L^{3} / T \quad \tau_{\mathrm{r}} \sim \tau_{0}\left(c L^{3}\right)^{2} .
$$

Finalement :

$$
f(\theta)=\frac{1}{Z} \exp \left[\lambda S+\left(\tau_{0}+\tau_{\mathrm{r}}\left(1-S^{2}\right) \dot{\gamma}\right) P_{2}(\cos \theta)\right] .
$$

Pour achever la description, $S$ est déterminé de façon self-consistente par l'équation :

$$
\| \begin{aligned}
& X=\lambda S+\tau_{0}+\tau_{\mathrm{r}}\left(1-S^{2}\right) \dot{\gamma} \\
& S=F(X)=\frac{\partial \ln Z}{\partial X}=-\frac{1}{2}+\frac{1}{2} X\left\{\frac{\exp \frac{3}{2} X}{\int_{0}^{1} \mathrm{~d} n \exp \frac{3}{2} X x^{2}}-1\right\} .
\end{aligned}
$$

A ce stade, deux remarques s'imposent :

- pour $c \gg c^{*}$, on peut le plus souvent négliger $\tau_{0}$ devant $\tau_{\mathrm{r}}\left(1-S^{2}\right)$;

- l'analyse de Doi et Edwards ne prend pas en compte la longueur finie des bâtons, qui commence juste à avoir une influence près de la transition nématique. Doi [7] a proposé d'interpoler $\tau_{\mathrm{r}}$ dans cette limite par :

$$
\tau_{\mathrm{r}}=\frac{\tau_{0}\left(c L^{3}\right)^{2}}{\left(\alpha-c / c_{\mathrm{n}}\right)^{2}}
$$

Nous ignorons cette complication (importante si on veut connaître la manière exacte dont la viscosité diverge à la transition nématique), qu'on peut d'ailleurs facilement inclure.

Sur les figures $9 a, 9 b, 9 c$ sont représentées les variations de $S$ en fonction de $\lambda=4,5 \frac{c}{c_{\mathrm{n}}}$ et $\tau_{\mathrm{r}} \dot{\gamma}$.

Pour $S$ fixé, donc $X$ fixé par l'équation (22a), la relation entre $\lambda$ et $\tau_{\mathrm{r}} \dot{\gamma}$ est linéaire. La surface $S\left(\lambda, \tau_{\mathrm{r}} \dot{\gamma}\right)$ est donc une surface réglée simple engendrée par les droites des plans $S=$ Cte s'appuyant sur les courbes $S(\lambda, 0)$ et $S\left(0, \tau_{\mathrm{r}} \dot{\gamma}\right)$. Il existe un domaine « biphasique " où la fonction $S(\dot{\gamma}, \lambda)$ est bivaluée. On a dessiné sur la figure $9 d$ la projection de ce domaine sur le plan $\lambda$, 

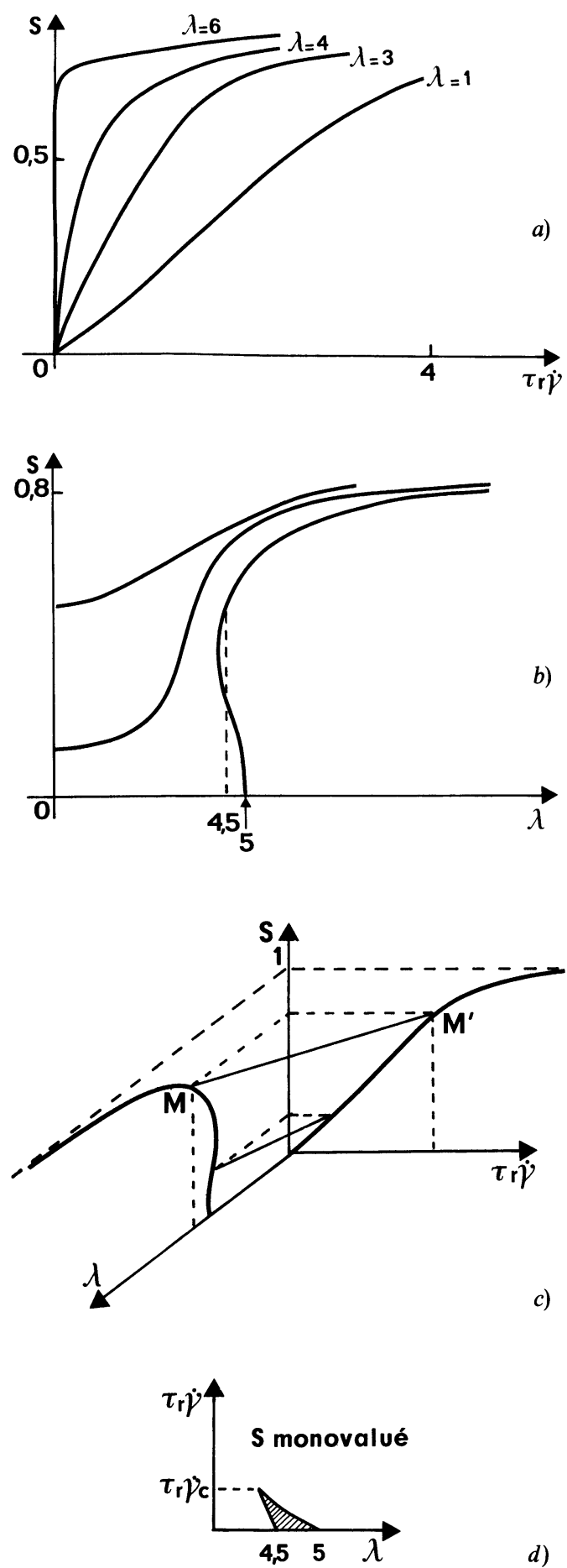

c)

d)

Fig. 9. - a) Allure du paramètre d'ordre de la solution de bâtonnets en fonction de $\tau_{\mathrm{r}} \dot{\gamma}$ à différentes concentrations. $b$ ) Paramètre d'ordre en fonction de $\lambda$ (concentration) pour $\tau_{\mathrm{r}} \dot{\gamma}$ constant (différent de $\dot{\gamma}$ constant). c) La surface réglée $S\left(\lambda, \tau_{\mathrm{r}} \dot{\gamma}\right)$ : paramètre d'ordre en fonction du taux de cisaillement et de la concentration. $d$ ) Domaine biphasique de la solution de bâtonnet, dans un écoulement orientationnel (donnée par l'enveloppe des projections des droites $\mathrm{MM}^{\prime}$ sur le plan $\left.\lambda, \tau_{\mathrm{r}} \dot{\gamma}\right)$.

[a) Order parameter of the rod solution expressed as a function of $\tau_{\mathrm{r}} \dot{\gamma}$ at different concentrations. b) Order parameter as function of $\lambda$ (concentration) for $\tau_{\mathrm{r}} \dot{\gamma}$ constant (different from $\dot{\gamma}$ constant). c) The ruled surface $S\left(\lambda, \tau_{\mathrm{r}} \dot{\gamma}\right)$ : order parameter as function of shear rate and concentration. $d$ ) Two phase region of the rod solution, in orientational flow (given by the envelope of projections of the lines MM on the plane $\left.\lambda, \tau_{\mathbf{r}} \dot{\gamma}\right)$.] $\tau_{\mathrm{r}} \dot{\gamma}$. La zone de transition nématique sous gradient élongationnel est à l'intérieur de ce domaine d'instabilité. Il existe un gradient critique au-delà duquel la transition n'existe plus.

On peut aussi forcer la transition nématique par un écoulement orientationnel. Ceci peut donner lieu à des effets spectaculaires dans une expérience de biréfringence d'écoulement.

En dessous du seuil nématique, nous pouvons écrire la réponse du système à un faible gradient, en linéarisant l'équation (22b) autour de $S=0$, il vient :

$$
S=F^{\prime}(0) \frac{\tau_{\mathrm{r}} \dot{\gamma}}{1-F^{\prime}(0) \lambda}=\frac{\tau_{\mathrm{r}} \dot{\gamma}}{5-\lambda} .
$$

$\lambda=\lambda^{* *}=5$ est la limite d'instabilité du système (dans l'approximation quadrupolaire). La susceptibilité augmente peu à la transition nématique ( $\lambda$ nématique $: 4,5)$ : les effets prétransitionnels sont peu importants. D'une manière générale, l'effet puissant de polarisation d'un écoulement à taux de cisaillement longitudinal esț encore amplifié dans les solutions semi-diluées à cause du ralentissement de la diffusion rotatoire.

3.1.2 Cas d'un écoulement non stationnaire. - Une étude précise devrait se faire à partir de l'équation de diffusion de Doi et Edwards. Nous proposons une approche plus simple à ce formidable problème : nous décrivons la compétition entre la polarisation par l'écoulement et la diffusion rotationnelle par une équation phénoménologique d'évolution des paramètres d'ordre que nous écrivons :

$$
\frac{\mathrm{d} S}{\mathrm{~d} t}=-\frac{-S}{\tau_{\mathrm{r}}\left(1-S^{2}\right)+\tau_{0}}+\dot{\gamma}(1-S) .
$$

Le sens physique de ces deux termes est clair. Les conclusions qu'on tire de cette équation est en accord qualitatif avec les études plus précises déjà effectuées :

- A l'équilibre, $S_{\text {eq }}$ est donné par

$$
\tau_{\mathrm{r}} \dot{\gamma}=\frac{S}{\left(1-S^{2}\right)(1+S)}
$$

L'allure de cette courbe est semblable à celle de la figure $9 a$.

- A gradient nul (expérience de relaxation de la biréfringence Kerr, par exemple) :

$$
\frac{\mathrm{d} S}{\mathrm{~d} t}=-\frac{S}{\tau_{\mathrm{r}}\left(1-S^{2}\right)}
$$

(on néglige le petit domaine où les molécules sont désenchevêtrées).

- A $t=0 ; S=1$ (par exemple) on a :

$$
-\log S+\frac{1}{2}(S-1)=\frac{t}{\tau_{\mathrm{r}}} \text {. }
$$


Aux temps courts si $S=1-\varepsilon$ on trouve :

$$
\varepsilon \sim\left(\frac{t}{\tau_{\mathrm{r}}}\right)^{1 / 2} .
$$

La décroissance initiale de $S$ est très rapide, comme l'ont décrit Doi et Edwards (avec le même exposant).

Aux temps longs, on retrouve une décroissance exponentielle de $S$, avec la constante de temps $\tau_{\mathrm{r}}$.

- A très fort gradient $\left(\tau_{\mathrm{r}} \dot{\gamma} \gg 1\right)$, on peut négliger le terme de relaxation $\mathrm{d} S / \mathrm{d} t=\dot{\gamma}(1-S)$ décrit l'orientation exponentielle et déterministe de la molécule dans un temps $\dot{\gamma}^{-1}$.

Si $S$ est proche de 1,

$$
S=\frac{1}{2}\left(3 \cos ^{2} \theta-1\right)=1-\frac{3}{4} \theta^{2}
$$

où $\theta$ est l'angle de la molécule avec l'écoulement.

$\mathrm{d} S / \mathrm{d} t=\dot{\gamma}(1-S)$ équivaut à $\dot{\theta}=-\gamma / 2 \theta$. C'est-àdire que $\theta$ décroît aussi exponentiellement avec une constante de temps de l'ordre de $\gamma^{-1}$ ce qui est cohérent avec l'équation mécanique des couples de friction.

$$
\begin{aligned}
T \tau \dot{\theta} \sim \frac{\mathrm{d}}{\mathrm{d} \theta}\left(T(\tau \dot{\gamma}) P_{2}(\cos \theta)\right) & \rightarrow \\
& \rightarrow \dot{\theta} \sim-\dot{\gamma} \theta \quad \text { (si } \theta \text { petit) } .
\end{aligned}
$$

(Les équations sont évidemment écrites à un facteur numérique près.)

3.2 COMPORTEMENT EN ÉCOULEMENT CONVERGENT. a) Caractéristiques de l'écoulement. - $\mathrm{Si} J_{\mathrm{s}}$ est le flux de solvant à travers le pore, il existe loin des pores et de la zone d'entrée (de rayon $\sim D, D<L$ ) un champ de vitesse radial $v_{\mathrm{r}}=J_{\mathrm{s}} / 4 \pi r^{2}$ (nous avons utilisé la conservation du courant) (voir Fig. 10) et en chaque point un gradient de vitesse :

$$
\dot{\gamma}=\frac{\mathrm{d} v_{\mathrm{r}}}{\mathrm{d} r}=\frac{J_{\mathrm{s}}}{2 \pi r^{2}}
$$

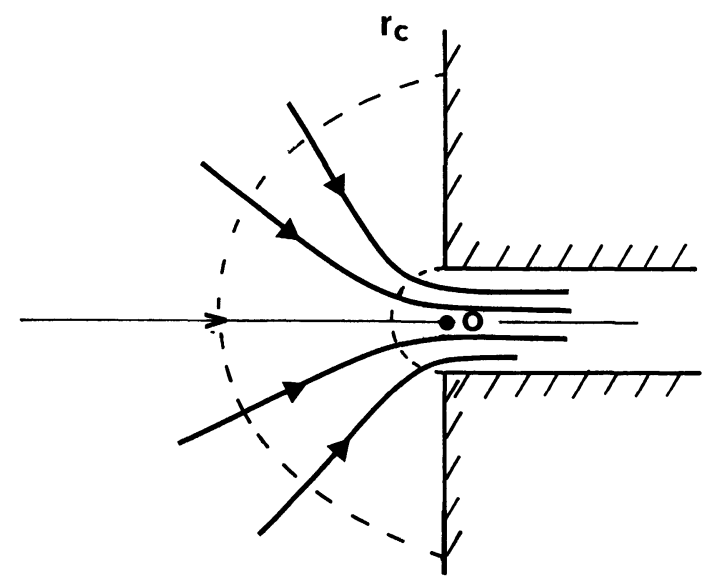

Fig. 10. - Ecoulement à l'entrée d'un pore.

[Flow at the entrance to a pore.]
Le temps typique de variation de ce gradient, en suivant une molécule entraînée par le fluide (nous négligeons la diffusion de translation) est :

$$
\dot{\gamma} \frac{\mathrm{d} \dot{\gamma}^{-1}}{\mathrm{~d} t}=\frac{\dot{\gamma}}{v_{\mathrm{r}}} \frac{\mathrm{d} \dot{\gamma}^{-1}}{\mathrm{~d} r} \sim \dot{\gamma}^{-1}
$$

Si $\tau$ est le temps caractéristique de la molécule dans la région de l'espace (pour les solutions diluées $\tau=\tau_{0}$, pour les solutions semi-diluées $\tau=\tau_{\mathbf{r}}$ ) où $\tau \dot{\gamma} \ll 1$ on aura une polarisation faible de quasi-équilibre; pour $\tau \dot{\gamma} \gg 1$ l'approximation quasi-stationnaire n'est plus valable et la polarisation est forte. Il se trouve que pour cet écoulement, les deux critères sont identiques. Le paramètre qui gouverne les phénomènes est donc la distance $r_{\mathrm{c}}$ telle que $\dot{\gamma}\left(r_{\mathrm{c}}\right) \tau \sim 1$ sont :

$$
r_{\mathrm{c}} \sim\left(J_{\mathrm{s}} \tau\right)^{1 / 3} \text {. }
$$

b) Entrée dans le pore. - Nous pouvons discuter maintenant l'orientation progressive des molécules dans l'écoulement et leur passage dans l'orifice du tube. Notre critère d'entrée est évidemment qu'une molécule ne peut pénétrer dans le pore si elle n'est pas suffisamment orientée (dans l'angle solide $\left.(D / L)^{2}\right)$ à une distance $L$ de l'orifice.

En écoulement lent $\left(J_{\mathrm{s}}\right.$ tel que $\left.r_{\mathrm{c}}<L\right)$ le gradient perturbe peu la solution. La majeure partie des bâtons qui se présente à l'entrée est mal orientée et rejetée. Seule une fraction de l'ordre de $(D / L)^{2}$ (coefficient de partage statique si $D<L$ et $c<c_{n}$ ) du flux de polymère à l'infini $\left(c_{\mathrm{p}} J_{\mathrm{s}}\right)$ passe dans le pore.

Ce cas est à opposer à celui des polymères flexibles : contrairement au bâton, leur probabilité d'entrée spontanée dans le pore est exponentiellement petite et il leur faut un certain flux critique pour être aspirés [19].

- $\mathrm{Si}$, au contraire, $J_{\mathrm{s}}$ est suffisamment grand pour que $r_{\mathrm{c}}$ soit supérieur à $L$, une molécule venant de l'infini va successivement se trouver sous deux régimes :

- un régime de liberté où la diffusion rotationnelle domine largement, et la molécule est peu orientée;

- et à partir de $r_{\mathrm{c}}$, un régime d'orientation forcée par l'écoulement.

La manière exacte dont on fait cette orientation est très sensible aux détails du modèle. Partons de l'équation déterministe valable à fort gradient pour des molécules très orientées :

$$
\begin{gathered}
\frac{\mathrm{d} \theta}{\mathrm{d} t}=-\alpha \dot{\gamma} \theta \quad \alpha=0(1) \\
v_{\mathrm{r}}=\frac{\mathrm{d} r}{\mathrm{~d} t}=-\frac{J_{\mathrm{s}}}{4 \pi r^{2}} ; \quad \frac{\mathrm{d} \theta}{\mathrm{d} t}=v_{\mathrm{r}} \frac{\mathrm{d} \theta}{\mathrm{d} r}
\end{gathered}
$$

donc

$$
\frac{\mathrm{d} \theta}{\mathrm{d} r}=+\alpha \frac{\dot{\gamma}(r)}{v(r)} \theta=2 \alpha \frac{\theta}{r},
$$

$\theta$ décroît en $r^{2 \alpha}$. 
Ce régime est équivalent au régime de déformation affine des polymères flexibles. On atteint rapidement le seuil où toutes les molécules pénètrent dans le pore. La plupart de ces molécules sont alors complètement orientées et vont être entraînées dans le tube sur une certaine distance avant que leur orientation ait atteint un régime stationnaire. Cette distance est le produit de la vitesse par le temps d'orientation dans le tube

$$
\Delta x=\tau_{D} v=\tau \frac{D^{2}}{L} \frac{J_{\mathrm{s}}}{D^{2}}=\frac{\tau J_{\mathrm{s}}}{L^{2}} .
$$

Dans des expériences où l'on désire éliminer les effets d'entrée, on devra prendre une longueur de pore très supérieure à cette distance de relaxation, et éventuellement tenir compte d'autres effets négligés ici : rétention possible des macromolécules dans les pores, accumulation de polymères à l'entrée du tube, formation de microgels bouchant les pores.

4. Conclusion. - Certaines des idées présentées ici sur les effets de déplétion près d'une paroi, sur l'interaction entre sphères dont les bâtons sont l'agent, sur les viscosités en milieu confiné et la rhéologie des solutions de bâtonnets demanderaient des développements théoriques plus précis. Leur seul mérite est de proposer certaines expériences à venir.

Nous pensons d'abord aux expériences de rhéologie qui paraissent les plus favorables :
- pour étudier précisément la réduction de viscosité des solutions de macromolécules rigides, longues et minces confinées dans des nucléopores. Les pores de petites dimensions (on sait maintenant réaliser des nucléopores de diamètre supérieure à $300 \AA$ ) ont deux avantages : les effets sont plus marqués, et ils permettent de travailler avec des molécules relativement plus courtes, donc moins flexibles;

- pour étudier les solutions semi-diluées en écoulement élongationnel. Les expériences de biréfringence d'écoulement sont alors un complément naturel. La difficulté vient des faibles gradients nécessaires pour faire apparaître les effets (à des concentrations telles que $\left(c / c^{*}\right)=10$ pour des molécules dont le coefficient de diffusion est $10^{3} \mathrm{~s}^{-1}$, les gradients doivent être de l'ordre de $10 \mathrm{~s}^{-1}$ ).

La mise en évidence des effets de floculation de microsphères induites par les bâtonnets durs serait également intéressante.

Dans bien des cas pratiques, notre modèle, qui inclut des effets uniquement stériques, et suppose des bâtons parfaitement rigides, tombe naturellement en défaut. Mais, il permet tout de même un premier inventaire qui pourra s'avérer utile.

Remerciements. - Je remercie enfin P. G. De Gennes et F. Brochard qui m'ont introduit à la physique de ces systèmes pour les discussions que nous avons eues à leur sujet.

\section{Bibliographie}

[1] ONSAGer, L., Ann. N. Y. Acad. Sci. 51 (1949) 627.

[2] Flory, P. J., Proc. R. Soc. A 234 (1956) 73.

[3] Straley, J. P., Mol. Cryst. Liq. Cryst. 22 (1973) 333.

[4] De Gennes, P. G., The Physics of liquid crystals (Oxford University Press) 1974.

[5] Bird, R., Hassager, O., Armstrong, R., Curtis, C., Dynamics of polymeric liquids, II (J. Wiley) 1977.

[6] Riseman, J., Kirkwood, J., J. Chem. Phys. 18 (1950) 512. Kirkwood, J., Auer, P., J. Chem. Phys. 19 (1951) 281.

[7] DoI, M., J. Physique 36 (1975) 607.

DoI, M., Edwards, S. F., J.C.S. Faraday Trans. II 74 (1958) 540.

DoI, M., Edwards, S. F., J.C.S. Faraday Trans. II 74 (1978) 918.

[8] De Gennes, P. G., Scaling concepts in polymer physics (Cornell Univ. Press) 1979.

[9] Maguire, J. F., McTague, J. P., Rondelez, F. (preprint).

[10] Chauveteau, G., C.R. Hebd. Séan. Acad. Sci. 288 (1975) 107.

Chauveteau, G., Communication au congrès de la Society of Rheology, 28.X-2.XI.79, Boston.

Chauveteau, G., à paraître dans $J$. Rheol.
[11] Joanny, J. F., Leibler, L., De Gennes, P. G., J. Polym. Sci. 17 (1979) 1073.

[12] IVEs, K. I. (Ed.), The scientific basic of flocculation (Sijthoff et Noordhoff) 1978.

[13] SCHAH, D., SCHECHTER, R., (Eds.), Improved oil recovery by surfactant and polymer flooding (Academic press) 1977.

[14] Taylor, G. I., Saffman, P. G., Proc. R. Soc. A 245 (1958) 312.

[15] Par ex. Kruyt, H. (Ed.), Colloid Science I. (Elsevier) 1952.

[16] De Gennes, P. G., C.R. Hebd. Séan. Acad. Sci., 24.IX.79, Série B, 105.

[17] Landau, L., Lifschitz, E., Théorie de l'élasticité (Mir) 1967, p. 202.

[18] Hess, S., Electro optics and dielectrics of macromolecules and colloids (1979), p. 321.

[19] DaOudi, S., Thèse de doctorat, Paris XI (1978). 